\title{
Stellar population astrophysics (SPA) with the TNG Characterization of the young open cluster ASCC 123*
}

\author{
A. Frasca ${ }^{1}$, J. Alonso-Santiago ${ }^{1}$, G. Catanzaro ${ }^{1}$, A. Bragaglia ${ }^{2}$, E. Carretta ${ }^{2}$, G. Casali $^{3}$, V. D’Orazi ${ }^{4}$, L. Magrini ${ }^{3}$, \\ G. Andreuzzi ${ }^{5,6}$, E. Oliva ${ }^{3}$, L. Origlia ${ }^{2}$, R. Sordo ${ }^{4}$, and A. Vallenari ${ }^{4}$
}

\author{
1 INAF-Osservatorio Astrofisico di Catania, Via S. Sofia 78, 95123 Catania, Italy \\ e-mail: antonio.frasca@inaf.it, afrasca@oact.inaf.it \\ 2 INAF-Osservatorio di Astrofisica e Scienza dello Spazio, Via P. Gobetti 93/3, 40129 Bologna, Italy \\ 3 INAF-Osservatorio Astrofisico di Arcetri, Largo E. Fermi 5, 50125 Firenze, Italy \\ 4 INAF-Osservatorio Astronomico di Padova, Vicolo dell'Osservatorio 5, 35122 Padova, Italy \\ 5 Fundación Galileo Galilei - INAF, Rambla José Ana Fernández Pérez 7, 38712 Breña Baja, Tenerife, Spain \\ 6 INAF-Osservatorio Astronomico di Roma, Via Frascati 33, 00078 Monte Porzio Catone, Italy
}

Received 13 September 2019 / Accepted 3 October 2019

\begin{abstract}
Star clusters are crucial to understanding stellar and Galactic evolution. ASCC 123 is a little-studied, nearby, and very sparse open cluster. We performed the first high-resolution spectroscopic study of this cluster in the framework of the Stellar Population Astrophysics (SPA) project with GIARPS at the TNG. We observed 17 stars, 5 of which turned out to be double-lined binaries. Three of the investigated sources were rejected as members on the basis of astrometry and lithium content. For the remaining single stars we derived the stellar parameters, extinction, radial, and projected rotational velocities, and chemical abundances for 21 species with atomic numbers up to 40 . From the analysis of single main-sequence stars we found an average extinction $A_{V} \simeq 0.13 \mathrm{mag}$ and a median radial velocity of about $-5.6 \mathrm{~km} \mathrm{~s}^{-1}$. The average metallicity we found for ASCC 123 is $[\mathrm{Fe} / \mathrm{H}] \simeq+0.14 \pm 0.04$, which is in line with that expected for its Galactocentric distance. The chemical composition is compatible with the Galactic trends in the solar neighborhood within the errors. From the lithium abundance and chromospheric $\mathrm{H} \alpha$ emission we found an age similar to that of the Pleiades, which agrees with that inferred from the Hertzsprung-Russell and color-magnitude diagrams.
\end{abstract}

Key words. stars: fundamental parameters - open clusters and associations: individual: ASCC 123 - stars: activity stars: abundances - binaries: spectroscopic

\section{Introduction}

Star clusters play a fundamental role in astronomy. Stars that formed in a cluster share a common origin, are located at the same distance (an approximation valid for all but the closest ones), and have an age and initial composition that are practically identical. However, small effects like atomic diffusion are observed in member stars of particularly well-studied clusters, such as M67 (e.g., Bertelli Motta et al. 2018). Therefore, they are the best laboratories to study stellar evolution and constrain theoretical models. Furthermore, the distribution of clusters of different age, mass, and distance allows us to study the Galactic disk. Open clusters (OCs) reveal the Galactic structure - they have been used since the 1960s to trace the spiral pattern of the Milky Way (see, e.g., Johnson et al. 1961; Janes \& Adler 1982; Junqueira et al. 2015) - and they permit us to study the disk's chemical evolution (e.g., Friel 1995).

\footnotetext{
* Based on observations made with the Italian Telescopio Nazionale Galileo (TNG) operated on the island of La Palma by the Fundación Galileo Galilei of the INAF (Istituto Nazionale di Astrofisica) at the Observatorio del Roque de los Muchachos. This study is part of the Large Program titled SPA - Stellar Population Astrophysics: the detailed, age-resolved chemistry of the Milky Way disk (PI: L. Origlia), granted observing time with HARPS-N and GIANO-B echelle spectrographs at the TNG.
}

Stellar Population Astrophysics (SPA) is an ongoing Large Program running on the Telescopio Nazionale Galileo (TNG) at La Palma. SPA is an ambitious project whose aim is to perform an age-resolved chemical map of the solar neighborhood and the Galactic thin disk. More than 500 representative stars (most of them located in clusters), covering different distances, ages and evolutionary stages, will be observed in the optical and nearinfrared (NIR) bands at high resolution by combining HARPS-N $(R=115000)$ and GIANO-B $(R=50000)$ spectrographs (see Origlia et al. 2019, for more details on SPA). The detailed chemical tagging carried out in this project will be combined with astrometric and photometric data obtained by the Gaia mission. This will provide many valuable clues to improve our understanding of possible trends, gradients, age-metallicity relations that allow us to constrain stellar evolution models and to determine in an accurate way the chemical evolution of the Galaxy. This work is one of the first papers of a series devoted to presenting the results of the SPA project.

ASCC 123 is a little-studied cluster located in the second Galactic quadrant $\left[\alpha(2000)=22^{\mathrm{h}} 42^{\mathrm{m}} 35^{\mathrm{s}}, \delta(2000)=+54^{\circ} 15^{\prime} 35^{\prime \prime}\right.$, $\ell=104.74^{\circ}, b=-4.00^{\circ}$. It was discovered by Kharchenko et al. (2005) when analyzing the HIPPARCos proper motions and $B V$ archival photometry contained in the All-Sky Compiled Catalog of 2.5 million stars (ASCC-2.5, Kharchenko 2001). They revealed the existence of a new cluster composed of 24 likely members 
spread in a large region on the sky with a radius of about $77^{\prime}$. They found a small reddening value, $E(B-V)=0.10$, and placed the cluster at a distance of $250 \mathrm{pc}$. They also estimated, with a large uncertainty, an age of $260 \mathrm{Myr}$ and a radial velocity of $\mathrm{RV}=-6.5 \mathrm{~km} \mathrm{~s}^{-1}$. Later, by combining astrometric data and NIR photometry from the PPMXL and 2MASS catalogs, Kharchenko et al. (2013) refined the reddening, $E(B-V)=0.15$, and the age, $\tau=155 \mathrm{Myr}$, of the cluster. Yen et al. (2018), based on the Gaia DR1/TGAS and HSOY data (Altmann et al. 2017), reanalyzed the parameters of the cluster and found values compatible with the previous ones: $E(B-V)=0.097, d=243.5 \mathrm{pc}$, and $\tau=130$ Myr. Finally, Cantat-Gaudin et al. (2018) from the Gaia DR2 astrometric data estimated a likely distance of $233.1 \pm 5.5 \mathrm{pc}$. These works compute the cluster extent from different approaches, which makes their comparison difficult. All estimates show a large and diffuse cluster with a core radius between $6-17^{\prime}$, which extends up to at least 60-78' . Depending on the radius considered, the cluster hosts from 24 to 121 stars as most likely members.

This is the first paper devoted exclusively to ASCC 123 to date. Until now, the cluster has been studied in an automatic way along with many more clusters. In this work we provide highresolution spectroscopy for 17 candidate members of this cluster for the first time with the aim of performing a full characterization in terms of atmospheric parameters, elemental abundance, rotation velocity, and activity level.

The paper is organized as follows. We present our data in Sect. 2, the cluster membership in Sect. 3, and color-magnitude diagrams (CMD) in Sect. 4. The data analysis and the main results are presented in Sect. 5 regarding the stellar parameters, and in Sect. 6 regarding the lithium content and chromospheric activity. The global properties of ASCC 123 are discussed in Sect. 7. In Sect. 8 we give a summary of the results of this work.

\section{Observations and data reduction}

In order to study the cluster we selected a series of representative stars among the brightest likely members according to the literature. The selection was made before the publication of the second Gaia data release. Therefore, as a first step we started with the list of 959 stars proposed by Kharchenko et al. (2005, 2013) as potential candidates. The selection was refined, rejecting several Kharchenko candidates and adding two stars, with the new Gaia DR2 data.

\subsection{Spectroscopic observations}

The observations were conducted from 18 to 22 August 2018 and on 11 August 2019 (see Table 1) with GIARPS (GIANOB \& HARPS-N; Claudi et al. 2017) at the $3.6 \mathrm{~m}$ TNG telescope located at El Roque de los Muchachos Observatory, in La Palma (Spain). GIARPS uses both the optical high-resolution spectrograph HARPS-N $(R \simeq 115000$, range $=0.39-0.68 \mu \mathrm{m}$, Cosentino et al. 2014) and the NIR spectrograph GIANO-B $(R \simeq 50000$, range $=0.97-2.45 \mu \mathrm{m}$; Oliva et al. 2012a, b; Origlia et al. 2014). The HARPS-N spectra were acquired with the second fiber on-sky. The spectra were acquired with total exposure times ranging from 1200 to $5700 \mathrm{~s}$, depending on the star brightness and sky conditions, with the aim of reaching a signal-tonoise ratio per pixel $S / N \geq 30$ at red wavelengths. Exposures longer than $1800 \mathrm{~s}$ were usually split in two or three to reduce the contamination of cosmic rays. The properties of the 17 stars observed at the TNG are listed in Table 2. In the present paper we only make use of HARPS-N spectra, which are best suited for the determination of stellar parameters and abundances of these relatively faint sources.
Table 1. Observation log.

\begin{tabular}{|c|c|c|c|c|c|}
\hline $\operatorname{ID}^{(a)}$ & Name & $\begin{array}{c}\text { Date_obs } \\
\text { yyyy-mm-dd }\end{array}$ & $\begin{array}{l}\text { UT_mid } \\
\text { hh:mm }\end{array}$ & $\begin{array}{l}t_{\exp } \\
(\mathrm{s})\end{array}$ & $S / N^{(b)}$ \\
\hline 39 & BD+54 2812 & 2018-08-19 & 02:19 & 3600 & 106 \\
\hline 56 & HD 235888 & 2018-08-19 & $05: 15$ & 1183 & 125 \\
\hline 56 & HD 235888 & 2018-08-21 & 04:15 & 1410 & 147 \\
\hline 214 & TYC 3983-2832-1 & $2018-08-20$ & $02: 36$ & 5700 & 90 \\
\hline 266 & BD+523260 & 2018-08-19 & $03: 28$ & 3600 & 111 \\
\hline 378 & TYC 3984-1809-1 & $2018-08-24$ & $00: 21$ & 2820 & 153 \\
\hline 435 & TYC 3988-1537-1 & $2018-08-23$ & 05:04 & 5700 & 81 \\
\hline 466 & HD 215178 & $2018-08-20$ & 01:15 & 3600 & 419 \\
\hline 490 & TYC 3984-1751-1 & $2018-08-23$ & 00:03 & 3600 & 70 \\
\hline 492 & TYC 3988-154-1 & $2018-08-23$ & $23: 19$ & 3600 & 109 \\
\hline 502 & TYC 3988-282-1 & $2018-08-20$ & $22: 22$ & 3600 & 75 \\
\hline 517 & TYC 3984-1588-1 & $2018-08-20$ & 04:44 & 5700 & 80 \\
\hline 554 & TYC 3984-1107-1 & $2018-08-23$ & 01:08 & 3600 & 132 \\
\hline 565 & TYC 3984-1717-1 & $2018-08-22$ & 01:47 & 3600 & 121 \\
\hline 708 & TYC 3984-277-1 & 2018-08-19 & 04:31 & 3600 & 153 \\
\hline 731 & TYC 3988-1310-1 & $2018-08-22$ & $02: 41$ & 1410 & 105 \\
\hline 731 & TYC 3988-1310-1 & $2018-08-23$ & 01:52 & 1380 & 110 \\
\hline $\mathrm{F} 1$ & $\mathrm{~J} 22452826+5347061$ & 2019-08-11 & $00: 50$ & 4800 & 36 \\
\hline $\mathrm{F} 2$ & $\mathrm{~J} 22311798+5502407$ & 2019-08-11 & $02: 23$ & 4800 & 34 \\
\hline
\end{tabular}

Notes. ${ }^{(a)}$ Sequential number from Kharchenko et al. (2005) based on right ascension. $\mathrm{F} 1$ and $\mathrm{F} 2$ are two additional candidates not included in Kharchenko et al. (2005). ${ }^{(b)}$ Signal-to-noise ratio per pixel at $6500 \AA$.

The HARPS-N spectra were reduced by the instrument Data Reduction Software pipeline. Radial velocities (RVs) were derived by this pipeline using the weighted cross-correlation function (CCF) method (Baranne et al. 1996; Pepe et al. 2002). However, we performed the CCF analysis again using synthetic template spectra and a broader RV window because most of our targets are binaries or rapidly rotating stars whose CCF peaks were not entirely covered by the RV range of the online CCF procedure. The RV values are listed in Table 3.

The telluric $\mathrm{H}_{2} \mathrm{O}$ lines at the $\mathrm{H} \alpha$ and $\mathrm{Na} \mathrm{I}_{2}$ wavelengths, as well as those of $\mathrm{O}_{2}$ at $6300 \AA$, were removed from the extracted HARPS-N spectra using an interactive procedure described by Frasca et al. (2000) and adopting telluric templates (spectra of hot, fast-rotating stars) acquired during the observing run.

\subsection{Archival data}

In order to complement our spectroscopic observations, and to characterize other likely cluster members, we resorted to archival data provided by some all-sky surveys. Specifically, we took $B V$ optical photometry from the APASS (Henden et al. 2015) and Tycho (ESA 1997) catalogs for the fainter and brighter sources, respectively. Values for the $I_{C}$ magnitudes were retrieved from the TASS catalog (Droege et al. 2006) and NIR $J H K_{s}$ magnitudes from the 2MASS catalog (Skrutskie et al. 2006).

In addition, we also took advantage of the great possibilities offered by the second Gaia data release (Gaia Collaboration 2018). We collected the high-quality photometric and astrometric data available in this release for the stars under study. The photometric data are listed in Table A.1, and the astrometric properties in Table 2.

\section{Cluster membership}

The disentangling of cluster members from field stars is not a trivial task and it is important to characterize the cluster itself. 
Table 2. Astrometric data of the stars in the field of ASCC 123 observed at the TNG.

\begin{tabular}{|c|c|c|c|c|c|c|c|c|c|c|c|}
\hline $\mathrm{ID}^{(a)}$ & Gaia DR2 ID & $\begin{array}{c}\text { RA } \\
(\mathrm{J} 2000) \\
\end{array}$ & $\begin{array}{c}\text { Dec } \\
(\mathrm{J} 2000) \\
\end{array}$ & $\begin{array}{c}r \\
\left({ }^{\prime}\right) \\
\end{array}$ & $P_{\mathrm{Kh} 05}{ }^{(b)}$ & $P_{\mathrm{Ca} 18}{ }^{(c)}$ & $\begin{array}{c}\varpi \\
\text { (mas) }\end{array}$ & $\begin{array}{c}\mu_{\alpha *} \\
\left(\operatorname{mas~yr}^{-1}\right)\end{array}$ & $\begin{array}{c}\mu_{\delta} \\
\left(\operatorname{mas~yr}^{-1}\right)\end{array}$ & $\begin{array}{c}G \\
(\mathrm{mag})\end{array}$ & $\begin{array}{c}B_{\mathrm{P}}-R_{\mathrm{P}} \\
(\mathrm{mag})\end{array}$ \\
\hline \multicolumn{12}{|c|}{ Members } \\
\hline 39 & 2003378188041736320 & 223513.26 & +544624.8 & 71.1 & 0.80 & 1.00 & 4.1879 & 12.303 & -0.578 & 10.352 & 0.624 \\
\hline 56 & 2003324655567305728 & 223536.32 & +543217.5 & 63.2 & 0.93 & & 4.0747 & 13.236 & -0.054 & 9.052 & 0.574 \\
\hline 214 & 2003023629898711680 & 223834.03 & +533508.7 & 53.8 & 0.69 & 1.00 & 4.2053 & 12.187 & -1.355 & 11.830 & 0.925 \\
\hline 266 & 2002270842392282496 & 223919.22 & +532916.5 & 54.6 & 0.83 & 1.00 & 4.1439 & 11.842 & -1.381 & 10.599 & 0.766 \\
\hline 378 & 2003132344109912832 & 224105.97 & +535904.2 & 21.0 & 0.40 & 0.70 & 4.2965 & 11.309 & -1.324 & 9.548 & 0.421 \\
\hline 435 & 2003443437181978240 & 224200.19 & +550058.5 & 45.7 & 0.99 & 1.00 & 4.2698 & 12.201 & -1.388 & 12.050 & 0.952 \\
\hline 466 & 2003171651651105664 & 224224.04 & +541454.2 & 1.7 & 0.61 & $\ldots$ & 4.2145 & 13.523 & -2.111 & 7.550 & 0.081 \\
\hline 492 & 2003173713235666560 & 224251.15 & +542353.5 & 8.6 & 0.81 & $\ldots$ & 4.7671 & 10.785 & 0.102 & 10.635 & 0.883 \\
\hline 517 & 2003161751738142464 & 224326.53 & +541158.4 & 8.4 & 0.70 & 1.00 & 4.2932 & 12.198 & -1.831 & 12.012 & 1.010 \\
\hline 554 & 2002409483936262016 & 224400.20 & +540838.1 & 14.3 & 0.95 & 1.00 & 4.1943 & 12.285 & -1.693 & 10.171 & 0.643 \\
\hline 708 & 2002765416464629504 & 224615.85 & +540136.6 & 35.2 & 0.75 & $\cdots$ & 4.6208 & 11.393 & -1.470 & 10.015 & 0.753 \\
\hline 731 & 2003554006818945280 & 224634.54 & +54 4605.2 & 46.2 & 0.97 & 1.00 & 4.3944 & 12.589 & -1.827 & 9.573 & 0.451 \\
\hline $\mathrm{F} 1$ & 2002337603362057088 & 224528.25 & +534706.1 & 38.2 & $\ldots$ & 1.00 & 4.2593 & 11.937 & -1.489 & 12.567 & 1.065 \\
\hline $\mathrm{F} 2$ & 2006435105245732480 & 223117.98 & +550240.7 & 108.6 & $\ldots$ & 1.00 & 4.1186 & 11.758 & -0.888 & 12.765 & 1.093 \\
\hline \multicolumn{12}{|c|}{ Non-members } \\
\hline 490 & 2002403436622121472 & 224249.48 & +540311.9 & 12.6 & 0.21 & & 3.6143 & 9.488 & -5.986 & 11.377 & 0.780 \\
\hline 502 & 2003178592318525696 & 224303.00 & +542253.8 & 8.4 & 0.79 & $\cdots$ & 4.5700 & 10.085 & -2.686 & 11.020 & 0.961 \\
\hline 565 & 2002120243658959488 & 224405.71 & +531356.3 & 63.1 & 0.79 & $\ldots$ & 4.5545 & 12.191 & 2.839 & 10.339 & 0.676 \\
\hline
\end{tabular}

Notes. ${ }^{(a)}$ Star identifier, as defined in Table 1. ${ }^{(b)}$ Membership probability according to Kharchenko et al. (2005), based on HIPPARCos astrometry. ${ }^{(c)}$ Membership probability according to Cantat-Gaudin et al. (2018), based on Gaia DR2 astrometry.

Based on the analysis of the proper motions from HIPPARcos, Kharchenko et al. (2005) identified 48 likely members to which they assigned a membership probability $(P)$ greater than or equal to 0.6 . These stars are distributed in a wide region of the sky centered at about $\alpha(2000)=22^{\mathrm{h}} 42^{\mathrm{m}} \cdot 5, \delta(2000)=+54^{\circ} 15^{\prime}$ with a radius of $\sim 75^{\prime}$. Cantat-Gaudin et al. (2018), taking advantage of the very precise astrometry provided by Gaia DR2, identified 55 members, considered as those stars with $P \geq 0.6$ in a field covering up to $140^{\prime}$ from the cluster center. For our targets the membership probabilities from the two works mentioned above are listed in Table 2. Seven stars in our sample, namely S 39, S 214, S 266, S 435, S 517, S 554, and S 731, are likely members according to both works. On the contrary, star 378 is quoted as a likely member only in Cantat-Gaudin et al. (2018), whereas six other objects have a membership probability $P_{\text {Kh05 }} \geq 0.6$ according to Kharchenko et al. (2005), but have no entry in the Cantat Gaudin catalog. Three of them (S 56, S 492, and S 708) turned out to be double-lined spectroscopic binaries (SB2s). Their position is close to the $5 \sigma$ contour in the proper motion diagram of Fig. 2. The stars labeled F1 and F2 are not included in the Kharchenko list, but they are very likely members $\left(P_{\mathrm{Ca} 18}=1\right)$ according to Cantat-Gaudin et al. (2018). Only S 490 is not a member in either work. Its astrometric parameters are clearly different from the average values of the cluster, as evaluated with the remaining stars. Moreover, as we show later (see Sect. 6), its very low lithium abundance is not compatible with the cluster age. In addition, despite the high value of membership probability $\left(P_{\mathrm{Kh} 05}=0.79\right)$, S 502 cannot be accepted as a cluster member because of its very low lithium abundance.

The spatial distribution of the stars investigated in the present paper is shown in Fig. 1, where the members according to Cantat-Gaudin et al. (2018) are also shown. The astrometric properties of our targets are displayed in the $\mu_{\alpha *}-\mu_{\delta}$ diagram (Fig. 2) along with the members according to CantatGaudin et al. (2018) and the stars in the field of ASCC 123 with magnitude $G \leq 15 \mathrm{mag}$. Cantat-Gaudin et al. (2018) computed
Table 3. Stellar parameters of the single/SB1 systems derived with the code ROTFIT.

\begin{tabular}{|c|c|c|c|c|c|c|c|c|c|c|}
\hline \multirow[t]{2}{*}{ ID } & $T_{\text {eff }}$ & Err & $\log g$ & Err & \multirow[t]{2}{*}{ SpT } & $\mathrm{RV}$ & Err & $v \sin i$ & Err & \multirow{2}{*}{$\begin{array}{l}A_{V}{ }^{(a)} \\
(\mathrm{mag})\end{array}$} \\
\hline & \multicolumn{2}{|c|}{$(\mathrm{K})$} & \multicolumn{2}{|c|}{$(\operatorname{dex})$} & & \multicolumn{2}{|c|}{$\left(\mathrm{km} \mathrm{s}^{-1}\right)$} & \multicolumn{2}{|c|}{$\left(\mathrm{km} \mathrm{s}^{-1}\right)$} & \\
\hline 39 & 6667 & 115 & 4.18 & 0.13 & $\mathrm{~F} 4 \mathrm{~V}$ & -6.36 & 3.31 & 49.1 & 1.9 & 0.01 \\
\hline 214 & 5804 & 87 & 4.41 & 0.12 & G1.5V & -6.39 & 3.92 & 100.9 & 3.0 & 0.23 \\
\hline 378 & 7636 & 293 & 4.18 & 0.14 & A7III & -5.6 & 10.9 & 71.1 & 5.8 & 0.18 \\
\hline 435 & 5758 & 79 & 4.39 & 0.12 & $\mathrm{G} 2.5 \mathrm{~V}$ & -4.41 & 0.26 & 11.6 & 0.7 & 0.14 \\
\hline 466 & 10500 & 250 & 4.00 & 0.20 & $\mathrm{~A} 0 \mathrm{~V}$ & -13.6 & 5.5 & 260.0 & 15.0 & 0.15 \\
\hline $490^{(*)}$ & 6128 & 113 & 3.95 & 0.11 & F8V & -15.80 & 0.20 & 2.9 & 1.8 & 0.06 \\
\hline 517 & 5784 & 81 & 4.39 & 0.12 & $\mathrm{G} 2 \mathrm{~V}$ & -7.03 & 5.59 & 83.6 & 1.9 & 0.36 \\
\hline 554 & 6871 & 152 & 4.14 & 0.13 & F4V & -5.21 & 6.41 & 81.8 & 3.3 & 0.20 \\
\hline $565^{(*)}$ & 6683 & 112 & 4.02 & 0.12 & $\mathrm{~F} 4 \mathrm{~V}$ & -1.85 & 2.45 & 23.8 & 0.8 & 0.15 \\
\hline 731 & 7355 & 274 & 4.00 & 0.20 & A7III & -7.54 & 8.41 & 68.9 & 7.0 & 0.07 \\
\hline $\mathrm{F} 1$ & 5263 & 92 & 4.55 & 0.10 & K0V & -4.35 & 0.13 & 6.6 & 0.6 & 0.00 \\
\hline $\mathrm{F} 2$ & 5237 & 77 & 4.54 & 0.10 & $\mathrm{~K} 1 \mathrm{~V}$ & -4.54 & 0.12 & 7.5 & 0.6 & 0.03 \\
\hline
\end{tabular}

Notes. ${ }^{(a)}$ Derived from the SED fitting. ${ }^{(*)}$ Non-member.

mean proper motions for ASCC 123 of $\overline{\mu_{\alpha *}}=12.093$ and $\overline{\mu_{\delta}}=$ $-1.407 \mathrm{mas} \mathrm{yr}^{-1}$, with standard deviations $\sigma_{\mu_{\alpha^{*}}}=0.473$ and $\sigma_{\mu_{\delta}}=0.437 \mathrm{mas} \mathrm{yr}^{-1}$. We adopted a $5 \sigma$ criterion, shown by the ellipse in Fig. 2, to confirm or discard candidate members. We found that, in addition to S 490, S 565 is also located well outside this boundary and should be considered as a nonmember. It has the largest RV among the single-lined objects $\left(\mathrm{RV}=-1.8 \mathrm{~km} \mathrm{~s}^{-1}\right)$, which supports its non-membership. However, as we see later, its $T_{\text {eff }}>6500 \mathrm{~K}$ does not permit us to use the lithium abundance as a further criterion for membership. The other non-member, S 502, is just out of the $5 \sigma$ boundary in the proper motion diagram.

In summary, among the 17 stars forming our sample, 14 are likely cluster members, whereas the other three, S 490, S 502, and S 565, should be considered non-members. To reinforce or reject the membership of our targets to ASCC 123, additional diagnostics based on high-resolution spectra, such as radial 


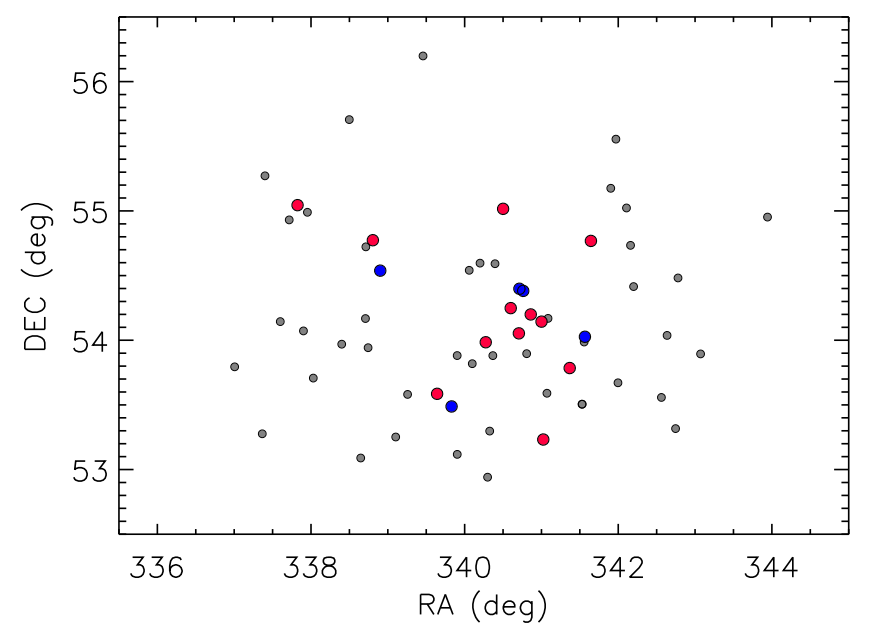

Fig. 1. Spatial distribution of the sources investigated in the present paper (big red dots for single stars, big blue dots for SB2 systems). The candidate members according to Cantat-Gaudin et al. (2018) are represented as small gray dots.

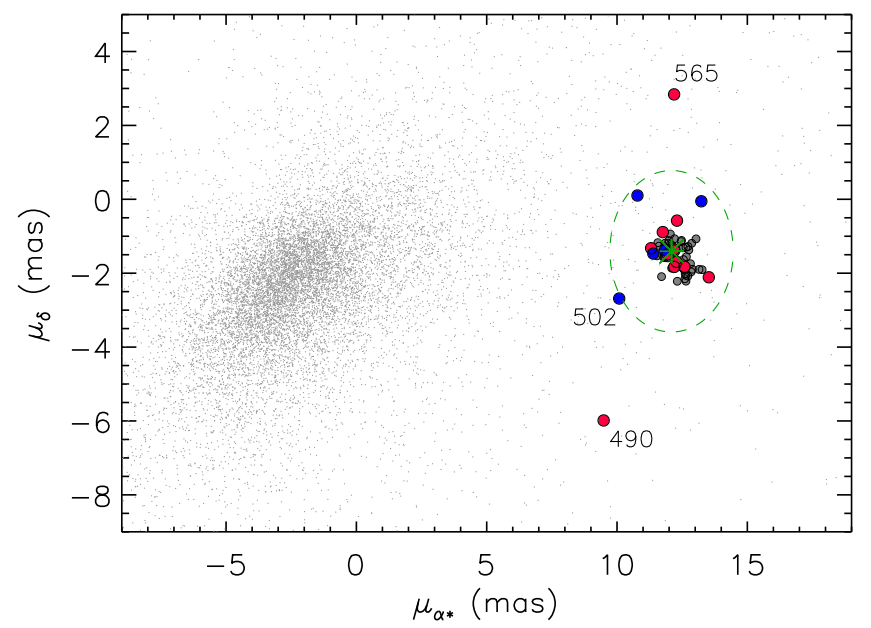

Fig. 2. Proper motion diagram of the sources with $G \leq 15 \mathrm{mag}$ in the ASCC 123 field (center coordinates $\alpha(2000)=22^{\mathrm{h}} 42^{\mathrm{m}} 35^{\mathrm{s}}$, $\delta(2000)=+54^{\circ} 15^{\prime} 35^{\prime \prime}$, radius $\left.75^{\prime}\right)$. The stars investigated in the present paper are indicated with big dots (red for single stars, blue for SB2 systems). The candidate members according to Cantat-Gaudin et al. (2018) are represented as small gray filled circles; the green asterisk denotes the average proper motion of ASCC 123 according to Cantat-Gaudin et al. (2018) and the dashed ellipse is the $5 \sigma$ contour. The three non-members that lie outside this locus are also labeled with their ID.

velocity, chromospheric activity, and lithium atmospheric content, must be used. This analysis is presented in Sects. 5 and 6.

\section{Color-magnitude diagrams and isochrone fitting}

The most common procedure to estimate the age of a star cluster is the so-called isochrone-fitting method. In a CMD several isochrones computed at different ages are drawn to find the one that best reproduces the location of the members in the diagram. While automated or Bayesian-based methods are well suited for most clusters, ASCC 123 was not included in Bossini et al. (2019) or in Monteiro \& Dias (2019). So, in order to ensure the reliability of the determination of the cluster parameters, we selected only those stars whose membership probability according to Cantat-Gaudin et al. (2018) is sufficiently high (i.e., $\left.P_{\mathrm{Ca} 18} \geq 0.75\right)$ as well as those members from Kharchenko et al. (2005) whose Gaia DR2 astrometry is compatible with the cluster. In Fig. 3 three CMDs are plotted in different photometric systems: optical $M_{V} /(B-V)_{0}$ (left panel), infrared $M_{K_{s}} /\left(J-K_{s}\right)_{0}$ (central panel), and Gaia magnitudes $G /\left(G_{\mathrm{BP}}-G_{\mathrm{RP}}\right)$ (right panel). In these CMDs we overplotted the targets observed in this work, distinguishing single stars from SB2 systems. Then we added PARSEC isochrones (Bressan et al. 2012) computed at the average metallicity found in this work, $[\mathrm{Fe} / \mathrm{H}]=+0.14$ (see Sect. 5.3). In the cases of the optical and infrared photometry, we corrected the magnitudes and colors for interstellar dust extinction adopting the average value found in the present paper for the single stars: $A_{V}=0.13$ (Sect. 5.4). In the Gaia CMD we instead preferred to display the reddened isochrones, computed with the calibrations of Maíz Apellániz \& Weiler (2018), since the dereddening of the Gaia photometry is not a trivial task. The distance adopted was that reported by Cantat-Gaudin et al. (2018) from Gaia parallaxes: $d=233$ pc.

In all three CMDs displayed in Fig. 3, three isochrones computed at 100, 155, and 250 Myr are plotted. The fit is quite good and, as expected, for the same color the SB2s are brighter than single stars. The absence of turn-off and evolved stars prevents an accurate determination of the cluster age; in the middle and upper main sequence (MS), where our members are located, isochrones in a wide age range behave in the same manner.

An interesting feature of these CMDs is the slight excess luminosity of the late-type members with respect to the model isochrone. This is best observed in the Gaia CMD (right panel in Fig. 3) because it includes the faintest and coolest members. This is similar to what has been observed for M dwarfs in the $\gamma$ Velorum (Jeffries et al. 2017) and Pleiades (Jackson et al. 2018) clusters. They ascribe this excess to stellar radii inflation driven by the strong magnetic fields of these young stars that inhibit convection and produce dark spots.

The three earliest members shown in the CMDs are S 866 (=HD 216057), S 466, and S 362 (=HD 214956). The first is a well-known Be star with an estimated spectral type B5Vne (Catanzaro 2013), while the other two objects are A0V stars (spectral types according to this work and Kharchenko et al. 2005, respectively). The presence of these early-type members suggests an age for the cluster slightly older than $100 \mathrm{Myr}$, which is in good agreement with the best-fitting isochrones shown in Fig. 3.

\section{Stellar parameters and abundances}

\subsection{Radial velocity}

The first step in our spectroscopic analysis is the measure of the radial velocity, which, as already mentioned in Sect. 2, is obtained by means of the cross-correlation of the HARPS-N spectra of our targets with synthetic templates. To do this we used the task FXCOR of the IRAF $^{1}$ package. The RV of the single-lined objects and of the components of SB2 systems is evaluated as the centroid of the CCF peak(s). The RVs for the single-lined members are listed in Table 3 and range from -7.5 to $-4.3 \mathrm{~km} \mathrm{~s}^{-1}$, if we exclude the hottest, fast-rotating star S 466 . We note that the errors are rather large, especially for the rapidly rotating stars. The median value of RV is $-5.6 \mathrm{~km} \mathrm{~s}^{-1}$, while the

1 IRAF is distributed by the National Optical Astronomy Observatory, which is operated by the Association of the Universities for Research in Astronomy, Inc. (AURA), under cooperative agreement with the National Science Foundation. 

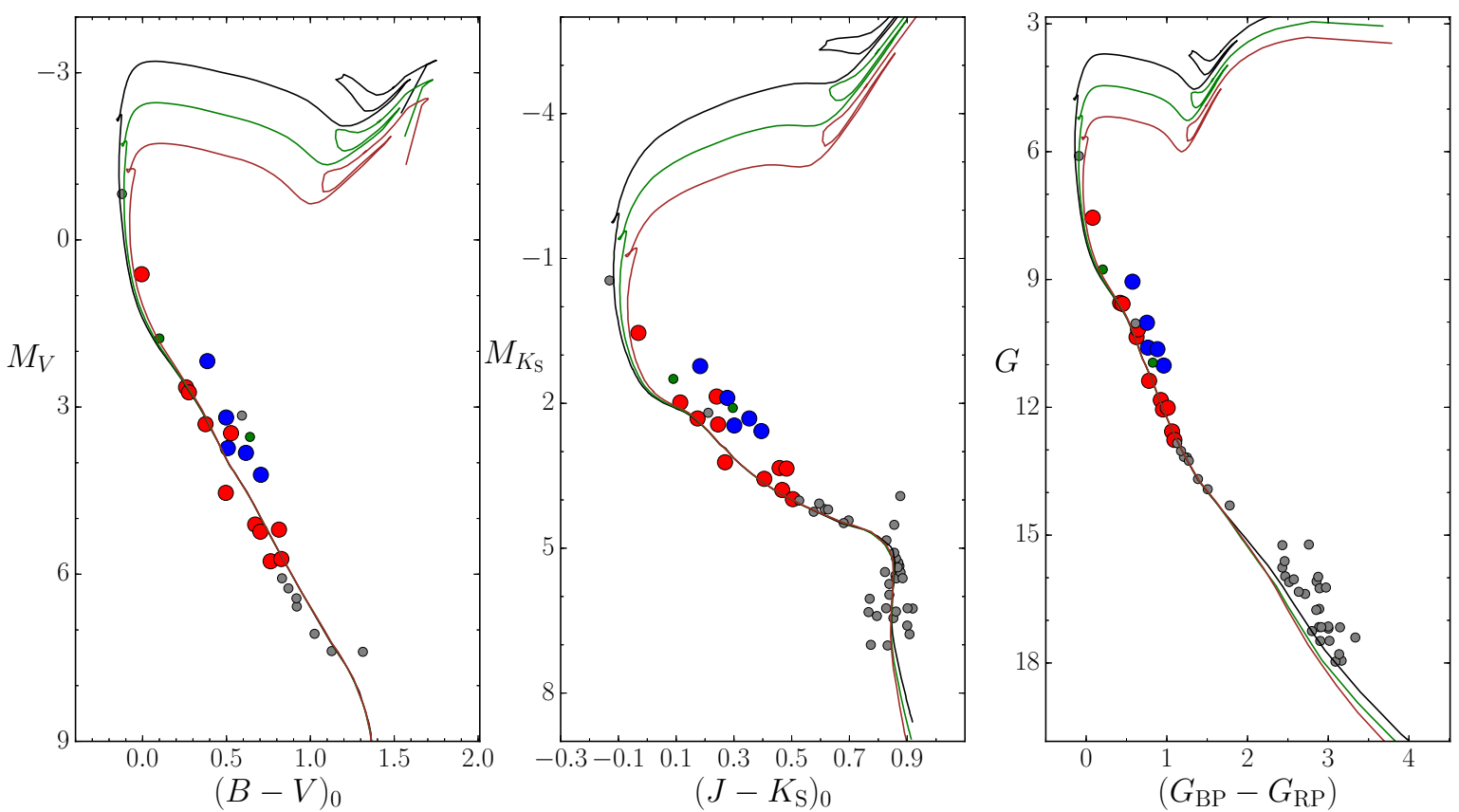

Fig. 3. Color-magnitude diagrams for likely members of ASCC 123 in three different photometric systems: left: $M_{V} /(B-V)_{0}$ from the APASS catalog; center: $M_{K_{\mathrm{s}}} /\left(J-K_{s}\right)_{0}$ (2MASS photometry); right: $G /\left(G_{\mathrm{BP}}-G_{\mathrm{RP}}\right)$ (Gaia DR2 data). Photometric data for likely members appear as small gray dots. Stars observed spectroscopically are represented as large red dots (single or possible SB1 objects) or blue dots (SB2). The solid lines represent the best-fitting PARSEC isochrones computed at $\tau=100 \mathrm{Myr}$ (black line), $\tau=155 \mathrm{Myr}$ (green line), and $\tau=250 \mathrm{Myr}$ (red line) assuming the metallicity and extinction found in the present work and the distance $d=233 \mathrm{pc}$ (Cantat-Gaudin et al. 2018).

Table 4. Stellar parameters of the components of SB2 systems derived with the code COMPO2.

\begin{tabular}{|c|c|c|c|c|c|c|c|c|c|c|c|c|}
\hline \multirow[t]{3}{*}{ ID } & \multirow{3}{*}{$\begin{array}{c}T_{\text {eff }} \\
(\mathrm{K}) \\
{[\mathrm{P} / \mathrm{S}]} \\
\end{array}$} & \multirow{3}{*}{$\begin{array}{c}\text { Err } \\
(\mathrm{K}) \\
{[\mathrm{P} / \mathrm{S}]} \\
\end{array}$} & \multirow{3}{*}{$\begin{array}{l}\log g \\
(\mathrm{dex}) \\
{[\mathrm{P} / \mathrm{S}]} \\
\end{array}$} & \multirow{3}{*}{$\begin{array}{c}\text { Err } \\
(\mathrm{dex}) \\
{[\mathrm{P} / \mathrm{S}]} \\
\end{array}$} & RV & Err & \multirow{3}{*}{$\begin{array}{c}\gamma \\
\left(\mathrm{km} \mathrm{s}^{-1}\right)\end{array}$} & \multirow[t]{3}{*}{$w_{4400}^{\mathrm{P}}$} & \multirow[t]{3}{*}{$w_{5500}^{\mathrm{P}}$} & \multirow[t]{3}{*}{$w_{6400}^{\mathrm{P}}$} & \multirow{3}{*}{$\begin{array}{l}\mathrm{SpT} \\
{[\mathrm{P} / \mathrm{S}]}\end{array}$} & \multirow{3}{*}{$\begin{array}{c}v \sin i \\
\left(\mathrm{~km} \mathrm{~s}^{-1}\right) \\
{[\mathrm{P} / \mathrm{S}]}\end{array}$} \\
\hline & & & & & \multicolumn{2}{|c|}{$\left(\mathrm{km} \mathrm{s}^{-1}\right)$} & & & & & & \\
\hline & & & & & {$[\mathrm{P} / \mathrm{S}]$} & {$[\mathrm{P} / \mathrm{S}]$} & & & & & & \\
\hline $56^{(a)}$ & $6670 / 6420$ & $150 / 250$ & $4.30 / 4.20$ & $0.15 / 0.20$ & $13.3 /-56.3$ & $4.5 / 13.3$ & $-18.5 \pm 6.8$ & $0.68 \pm 0.12$ & $0.66 \pm 0.10$ & & F2V/F7IV & $50 / 70$ \\
\hline 266 & $6094 / 5550$ & $64 / 209$ & $4.15 / 4.26$ & $0.11 / 0.22$ & $-78.3 / 54.7$ & $1.4 / 0.6$ & $-20.2 \pm 1.3$ & $0.79 \pm 0.03$ & $0.75 \pm 0.03$ & $0.74 \pm 0.04$ & F8V/G3V & $10 / 5$ \\
\hline 492 & $6075 / 5560$ & $59 / 168$ & $4.24 / 4.32$ & $0.06 / 0.17$ & $-26.2 / 12.9$ & $0.9 / 1.8$ & $-8.2 \pm 1.0$ & $0.69 \pm 0.03$ & $0.67 \pm 0.02$ & $0.65 \pm 0.04$ & F9V/G7V & $12 / 12$ \\
\hline $502^{(*)}$ & $5730 / 5628$ & $111 / 152$ & $4.32 / 4.36$ & $0.11 / 0.13$ & $-19.7 / 10.8$ & $0.5 / 0.6$ & $-4.7 \pm 0.4$ & $0.55 \pm 0.02$ & $0.54 \pm 0.02$ & $0.53 \pm 0.03$ & $\mathrm{G} 2 \mathrm{~V} / \mathrm{G} 2 \mathrm{~V}$ & $5 / 5$ \\
\hline 708 & $6114 / 6099$ & $81 / 183$ & $4.12 / 4.18$ & $0.16 / 0.19$ & $-25.7 / 34.6$ & $0.7 / 5.6$ & $4.0 \pm 2.8$ & $0.54 \pm 0.06$ & $0.53 \pm 0.05$ & $0.50 \pm 0.05$ & F8IV/F8IV & $10 / 50$ \\
\hline
\end{tabular}

Notes. ${ }^{(a)}$ Uncertain parameters due to the high $v \sin i$ values and the strong line blend. ${ }^{(*)}$ Non-member.

weighted average is $-4.5 \mathrm{~km} \mathrm{~s}^{-1}$. The values of RV for the components of SB2 systems are listed in Table 4.

\subsection{Atmospheric parameters}

We adopted the code ROTFIT (Frasca et al. 2006) to derive the basic atmospheric parameters (APs) for the stars with singlelined HARPS-N spectra (either single stars or SB1 systems). This code also allows us to measure the projected rotational velocity $(v \sin i)$ and to perform an MK spectral classification. ROTFIT uses a grid of template spectra and is based on a $\chi^{2}$ minimization of the difference observed-template in selected spectral regions. The grid of templates is composed of highresolution spectra of real stars with well-known APs, which were retrieved from the ELODIE archive. This grid is the same as that adopted in the Gaia-ESO Survey by the Catania (OACT) analysis node (see, e.g., Smiljanic et al. 2014; Frasca et al. 2015). When using this grid, the HARPS-N spectra of the targets are degraded to the resolution of ELODIE $(R=42000)$, which is fully suitable for our purposes, also because of the large rotational velocities. The target spectra were also resam- pled on the ELODIE spectral points $(\Delta \lambda=0.05 \AA)$. This also has the advantage of improving the $\mathrm{S} / \mathrm{N}$ of the spectra to be analyzed.

Each template is aligned in wavelength with the target spectrum thanks to the CCF in a suitable spectral region. After being aligned with the target spectrum, each template is broadened by convolution with a rotational profile of increasing $v \sin i$ until a minimum $\chi^{2}$ is attained. The weighted average of the parameters of the ten best templates is taken for each of the analyzed regions.

We analyzed 28 spectral segments of $100 \AA$ each from $4000 \AA$ to $6800 \AA$, which is the range covered by the ELODIE templates and corresponds to the portion of HARPS-N spectra where the $\mathrm{S} / \mathrm{N}$ is the best. The core of Balmer lines and the regions severely affected by telluric absorption were disregarded. The final parameters were obtained by averaging the results of the individual segments, weighting them according to the $\chi^{2}$ and the amount of information contained in each segment, which is evaluated as the total line absorption, $f_{i}=\int\left(F_{\lambda} / F_{\mathrm{C}}-1\right) \mathrm{d} \lambda$, where $F_{\lambda} / F_{\mathrm{C}}$ is the continuum-normalized spectrum in the $i$ th spectral segment. 

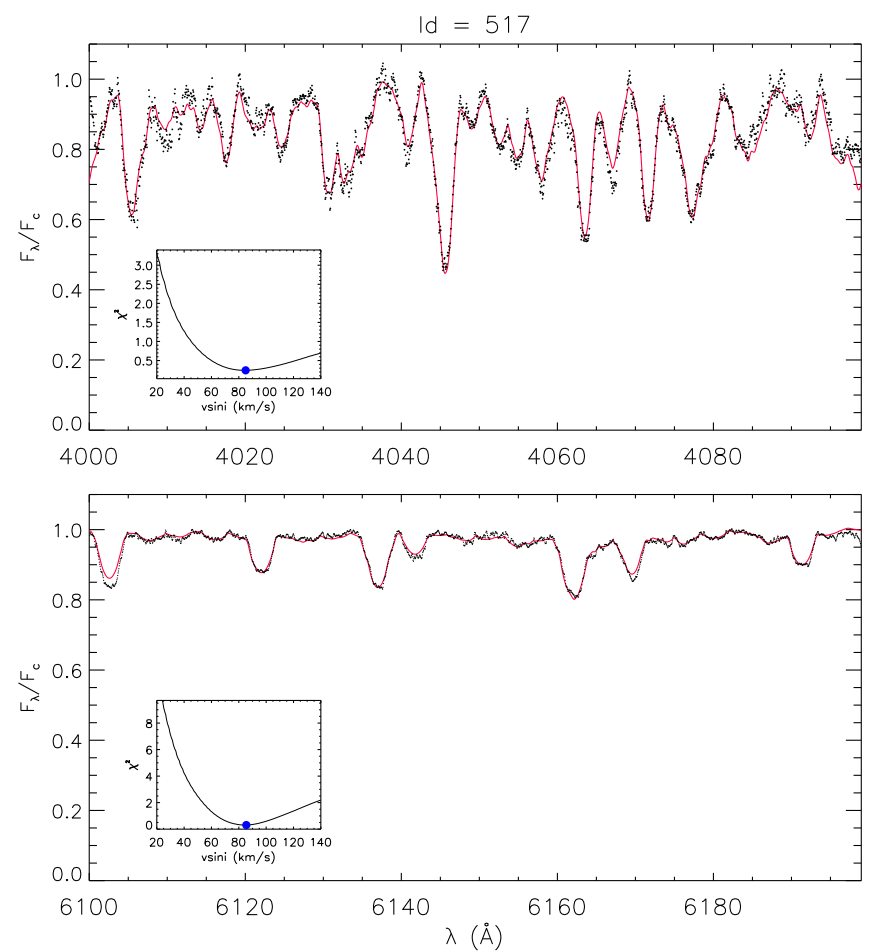

Fig. 4. Observed HARPS-N spectrum of S 517 (black dots) in the $\lambda 4000 \AA$ (upper panel) and $\lambda 6100 \AA$ (lower panel) spectral regions. In each panel the template spectrum broadened at the $v \sin i$ of the target is overplotted with a full red line. The insets show the $\chi^{2}$ of the fit as a function of the $v \sin i$.

An example of the application of the code ROTFIT to two spectral segments of S 517 is shown in Fig. 4, where the observed and template spectra are displayed with black dots and a red line, respectively. In the same figure, the $\chi^{2}$ as a function of $v \sin i$ is plotted in the insets.

The results of the analysis for the spectra of single/SB1 systems are summarized in Table 3.

Among the 17 observed targets we found five spectroscopic double-lined (SB2) systems. For four of them the spectral lines (and the peaks of the CCFs) are sufficiently separated in wavelength to allow us to meaningfully infer the stellar parameters of the components of these systems from the analysis of their spectra. To this aim we used COMPO2, a code developed in IDL ${ }^{2}$ environment by Frasca et al. (2006), which, like ROTFIT, was adapted to the HARPS-N spectra. COMPO2 works in a similar way to ROTFIT: it adopts a grid of templates (ELODIE spectra of real stars) to reproduce the observed composite spectrum, which is split into 28 segments of $100 \AA$ each that are independently analyzed. In this case the projected rotation velocities of the two components, $v \sin i_{1}$ and $v \sin i_{2}$, are not free parameters, but are computed from the full width at half maximum (FWHM) of the peaks of the CCF. The RV separation of the two components is derived from the centroids of the two CCF peaks. The continuum flux ratio (i.e., the flux contribution of the primary component in units of the continuum, $\left.w^{\mathrm{P}}\right)$ is an adjustable parameter. An example of the application of COMPO2 is shown in Fig. 5 for two spectral segments, one around the $\mathrm{Mg} \mathrm{I}$ b triplet and the other centered at $6440 \AA$, in which the lines of the two components are clearly distinguishable. 2 IDL (Interactive Data Language) is a registered trademark of Harris
Corporation.
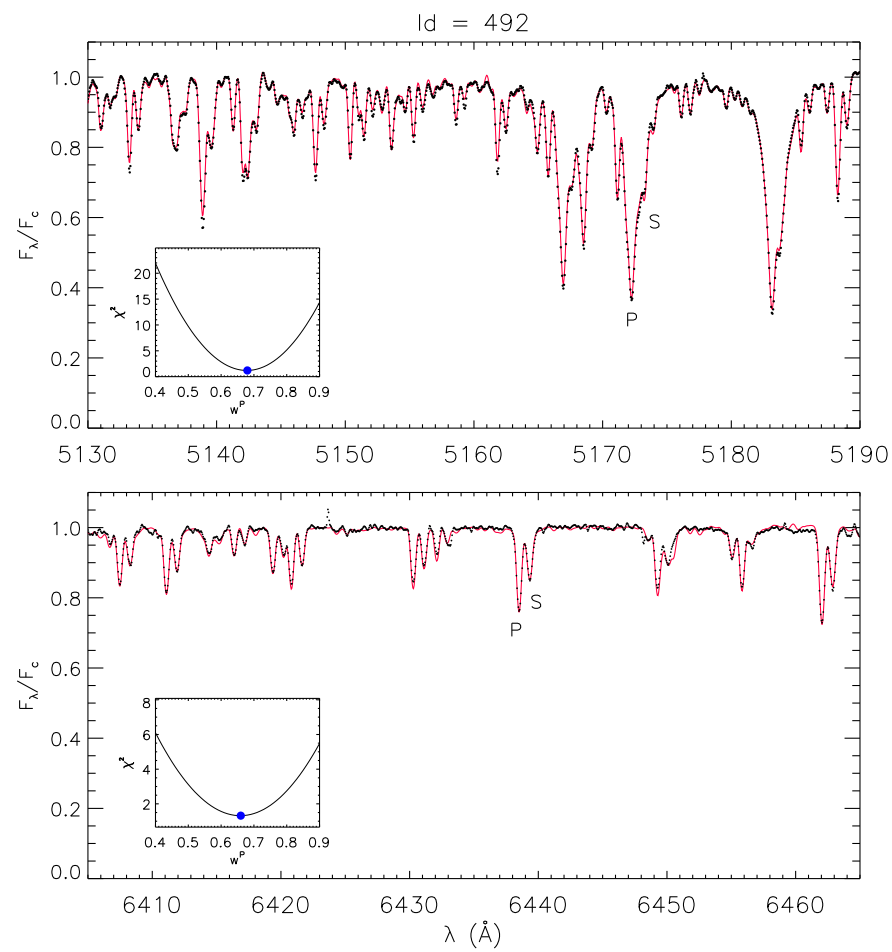

Fig. 5. Observed HARPS-N spectrum of the SB2 system S 492 (black dots) around the MgIb lines ( $\lambda 5160 \AA$, upper panel) and around $\lambda 6440 \AA$ (lower panel). In each panel the synthetic spectrum, which is the weighted sum of two standard star spectra mimicking the primary and secondary component of S 492, is overplotted with a full red line. The insets show the $\chi^{2}$ of the fit as a function of the contribution of the primary spectrum, $w^{\mathrm{P}}$.
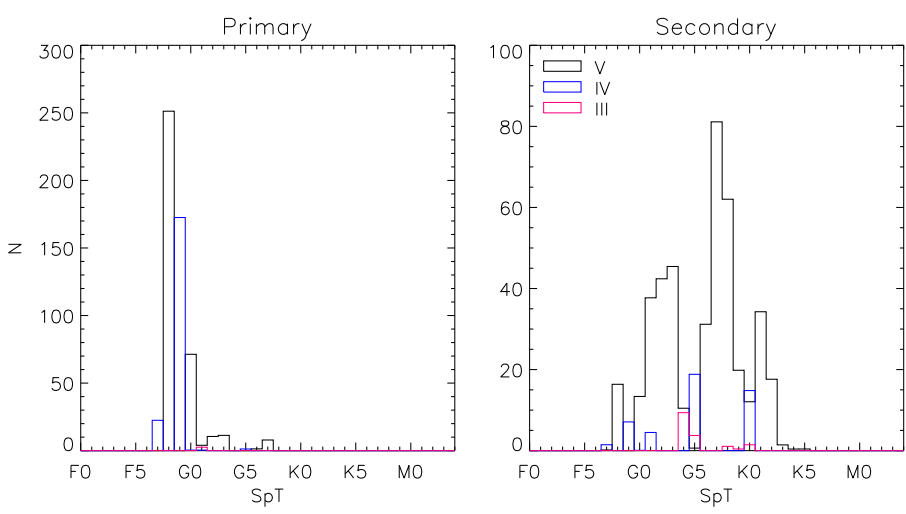

Fig. 6. Distribution of spectral types for the components of S 492.

To evaluate the APs we kept only the best 100 combinations (in terms of minimum $\chi^{2}$ ) of primary and secondary spectra per each spectral segment. The results for the individual segments were weighted as explained above for ROTFIT to calculate the average APs, which are listed in Table 4. The flux contribution was evaluated at three wavelengths (4400 $\AA$, $5500 \AA$, and $6400 \AA$ ) using only the spectral segments around these wavelengths. The relative contribution of the two components changes appreciably from blue to red wavelengths only if they have a very different $T_{\text {eff }}$. The spectral types (SpT) of the components are taken as the mode of the spectral-type distributions (see Fig. 6 for an example).

The system $\mathrm{S} 56$ displays a complex spectral and CCF behavior, which is similar to an SB2 with two fast-rotating stars 
( $v \sin i_{1} \sim 50 \mathrm{~km} \mathrm{~s}^{-1}, v \sin i_{2} \sim 70 \mathrm{~km} \mathrm{~s}^{-1}$ ) with blended lines in both spectra. We tried a determination of the stellar parameters of the two components with COMPO2 and obtained a decent fit of the observed spectrum in several spectral regions. However, the stellar parameters, especially those of the fainter, fasterrotating secondary, should be considered as indicative only.

For the SB2 systems we also evaluated the systemic velocity $\gamma$. In an ideal case of two components with the same mass, $\gamma$ is the mean of the radial velocities, $\gamma=\left(\mathrm{RV}_{1}+\mathrm{RV}_{2}\right) / 2$. In general, the systemic velocity can be calculated as

$\gamma=\mathrm{RV}_{1}+\frac{\left(\mathrm{RV}_{2}-\mathrm{RV}_{1}\right)}{\frac{M_{1}}{M_{2}}+1}$,

where $\mathrm{RV}_{1}$ and $\mathrm{RV}_{2}$ are the radial velocities and $M_{1}$ and $M_{2}$ the masses of the two components.

As we have only one or two spectra per each system, we cannot derive the mass ratio, $M_{1} / M_{2}$, which can only be derived by the solution of a full RV curve. Therefore, $M_{1} / M_{2}$ must be estimated by the spectra of these systems. Assuming that all the components are on the MS (which is probably not valid for S 56), we can use a mass-luminosity relation to estimate the mass ratio. To this end, we adopted the relation proposed by Eker et al. (2015, Table 3) for intermediate-mass stars, $\log L=$ $4.328 \times \log M-0.002$, from which we get

$\frac{M_{1}}{M_{2}}=\left(\frac{L_{1}}{L_{2}}\right)^{0.23}$.

The luminosity ratio $\frac{L_{1}}{L_{2}}$ was approximated by the ratio of flux contributions at $5500 \AA$ (reported in Table 4). The error of $\gamma$ was calculated propagating the errors of $\mathrm{RV}_{1}, \mathrm{RV}_{2}$, and $w_{5500}^{\mathrm{P}}$ through Eqs. (1) and (2). The values of $\gamma$ are also listed in Table 4 along with their errors.

The $\gamma$ values span a range similar to that of the RVs for the single-lined objects, with the exception, perhaps, of S 708 and $\mathrm{S} 266$. However, repeated observations are needed to get more accurate values of $\gamma$.

\subsection{Elemental abundances}

We also analyzed the HARPS-N spectra of the single-lined targets by means of a spectral synthesis approach (e.g., Catanzaro et al. 2011, 2013). For this task we did not degrade the spectra but maintained their original resolution $R=115000$. We used the ATLAS9 code (Kurucz 1993a,b) to compute local thermodynamic equilibrium (LTE) atmospheric models and SYNTHE (Kurucz \& Avrett 1981) to produce synthetic spectra that include both the instrumental and rotational broadening.

As a first step, we used the APs obtained with ROTFIT, but performed a check by letting them vary and found the best values by minimizing the $\chi^{2}$ of the difference observed-synthetic. We always found values consistent with those of ROTFIT within the errors. We therefore adopted the ROTFIT atmospheric parameters listed in Table 3 and used them for the subsequent analysis.

We then derived the abundances of elements of atomic number up to 40. In particular, we analyzed independently 39 spectral segments of $50 \AA$ in width between 4400 and $6800 \AA$. Ad hoc routines written in IDL allowed us to find the best solution by $\chi^{2}$ minimization. The elemental abundances, expressed in the standard notation $A(X)=\log [n(X) / n(H)]+12$, are listed in Table A.2. The weighted-averaged abundances for the cluster (considering only the confirmed members) are listed in Table 5 along with the standard error of the weighted mean. In this table
Table 5. Average elemental abundances for ASCC 123 and for the Sun derived in the present work.

\begin{tabular}{lcc}
\hline \hline Element & ASCC 123 & Sun $^{(a)}$ \\
\hline $\mathrm{C}$ & $8.41 \pm 0.06$ & $8.54 \pm 0.04$ \\
$\mathrm{O}$ & $9.09 \pm 0.20$ & $8.80 \pm 0.01$ \\
$\mathrm{Na}$ & $6.13 \pm 0.08$ & $6.17 \pm 0.14$ \\
$\mathrm{Mg}$ & $7.71 \pm 0.04$ & $7.70 \pm 0.10$ \\
$\mathrm{Al}$ & $6.47 \pm 0.06$ & $6.55 \pm 0.15$ \\
$\mathrm{Si}$ & $7.52 \pm 0.08$ & $7.50 \pm 0.08$ \\
$\mathrm{~S}$ & $7.42 \pm 0.05$ & $7.33 \pm 0.10$ \\
$\mathrm{Ca}$ & $6.60 \pm 0.04$ & $6.32 \pm 0.09$ \\
$\mathrm{Sc}$ & $3.52 \pm 0.04$ & $3.14 \pm 0.13$ \\
$\mathrm{Ti}$ & $4.99 \pm 0.07$ & $4.86 \pm 0.12$ \\
$\mathrm{~V}$ & $4.50 \pm 0.14$ & $3.96 \pm 0.10$ \\
$\mathrm{Cr}$ & $5.77 \pm 0.04$ & $5.57 \pm 0.15$ \\
$\mathrm{Mn}$ & $5.56 \pm 0.05$ & $5.35 \pm 0.15$ \\
$\mathrm{Fe}$ & $7.59 \pm 0.04$ & $7.45 \pm 0.08$ \\
$\mathrm{Co}$ & $5.35 \pm 0.18$ & $4.93 \pm 0.05$ \\
$\mathrm{Ni}$ & $6.29 \pm 0.06$ & $6.16 \pm 0.10$ \\
$\mathrm{Cu}$ & $4.44 \pm 0.10$ & $4.26 \pm 0.10$ \\
$\mathrm{Zn}$ & $4.44 \pm 0.08$ & $4.53 \pm 0.09$ \\
$\mathrm{Sr}$ & $3.55 \pm 0.08$ & $2.95 \pm 0.15$ \\
$\mathrm{Y}$ & $2.66 \pm 0.16$ & $2.25 \pm 0.04$ \\
$\mathrm{Zr}$ & $3.16 \pm 0.08$ & $2.65 \pm 0.14$ \\
\hline
\end{tabular}

Notes. The values are expressed according to the standard notation $A(X)=\log [n(X) / n(H)]+12 .{ }^{(a)}$ Derived from a HARPS-N spectrum of Ganymede.

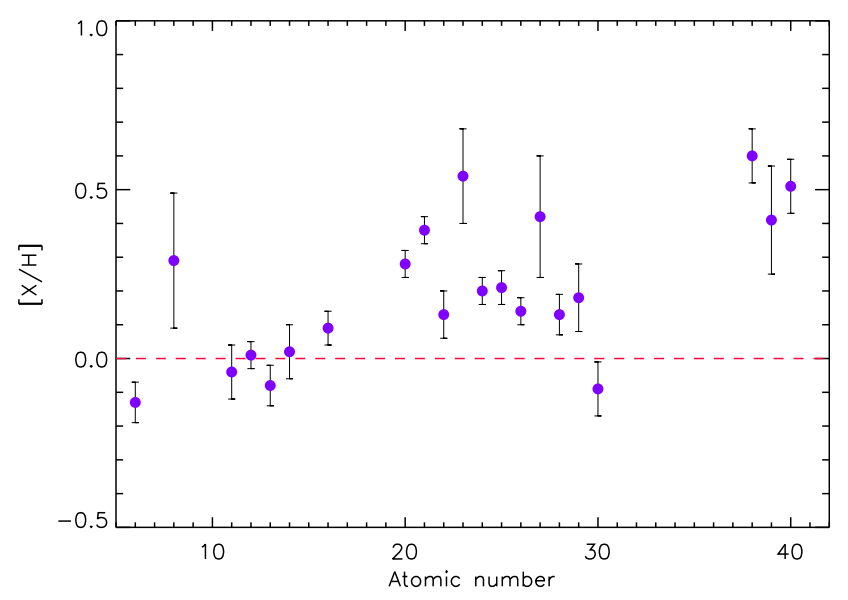

Fig. 7. Chemical pattern for ASCC 123. The horizontal dashed line corresponds to the solar abundances listed in Table 5.

we also give the abundances we derived for the Sun, applying the same procedure to a HARPS-N spectrum of Ganymede acquired in the framework of the Global Architecture of Planetary Systems (GAPS; Covino et al. 2013).

The pattern of chemical abundances for ASCC 123 is shown in Fig. 7, where we used as reference the solar abundances derived in the present work (Table 5). We note an average overabundance of $\sim 0.2$ dex with respect to solar values for the ironpeak elements. In particular, the iron abundance for the cluster is $[\mathrm{Fe} / \mathrm{H}]=+0.14 \pm 0.04$. The heavy elements $(\mathrm{Sr}, \mathrm{Y}$, and $\mathrm{Zr}$ ) are overabundant with respect to the Sun by about 0.5 dex.

The chemical pattern for the individual stars in the field of ASCC 123 is displayed in Fig. A.1. As can be seen, the nonmember S 490 shows all the elements that are underabundant 


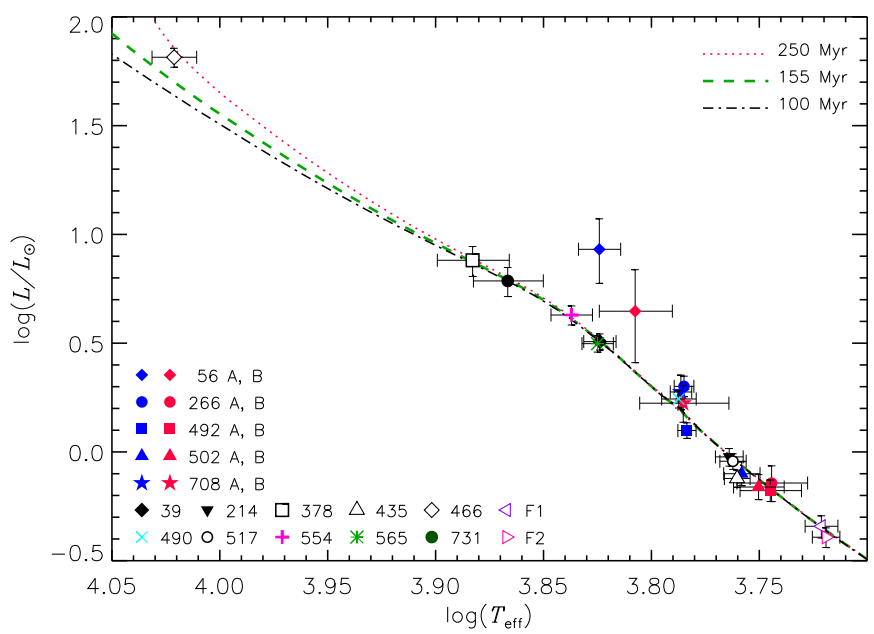

Fig. 8. HR diagram. The stars are coded with different symbols and colors. PARSEC isochrones at 100,155 , and $250 \mathrm{Myr}$ for models with $Z=0.021([\mathrm{Fe} / \mathrm{H}]=+0.14)$ are overlaid.

compared to the Sun, with an average value of $\sim-0.3$ dex. The other likely non-member S 565 displays a solar chemical abundance.

These results are discussed in more detail in Sect. 7.

\subsection{Spectral energy distributions and the Hertzsprung-Russell diagram}

For the single stars, we used the spectral energy distribution (SED) to evaluate the interstellar extinction, $A_{V}$, and to derive the luminosities. The SEDs were built with optical and NIR photometric data available in the literature and are listed in Table A.1.

The SEDs were fitted with NextGen low-resolution synthetic spectra (Hauschildt et al. 1999). For each target, we adopted the Gaia DR2 parallax and kept $T_{\text {eff }}$ and $\log g$ fixed to the values derived with ROTFIT (Table 3), while the stellar radius, $R_{*}$, and $A_{V}$ were set as free parameters. The best values of $R_{*}$ and $A_{V}$ were found by $\chi^{2}$ minimization. The luminosity was calculated as $L=4 \pi R_{*}^{2} \sigma T_{\text {eff }}^{4}$.

We found values of $A_{V}$ in the range $0.00-0.36 \mathrm{mag}$ (see Table 3 ) with an average of $0.13 \mathrm{mag}$. Assuming a typical value $R_{V}=3.1$ for the total-to-selective extinction, we obtain a reddening $E(B-V) \simeq 0.04 \mathrm{mag}$, which is lower than the value of $0.15 \mathrm{mag}$ reported by Kharchenko et al. (2013) for this cluster.

We derived the luminosities of the components of the SB2 systems from the combined $V$ magnitude listed in Table A.1 and the luminosity ratio at $5500 \AA$ stemming from $w_{5500}^{\mathrm{P}}$ (Table 4). The values of $V$ were corrected for the extinction $\left(A_{V}=\right.$ $0.13 \mathrm{mag}$ ) and were used to calculate the absolute magnitudes with the Gaia distances. The bolometric correction of Pecaut \& Mamajek (2013) was applied and the bolometric magnitude of the Sun, $M_{\text {bol }}^{\odot}=4.64 \mathrm{mag}$ (Cox 2000), was used to express the stellar luminosity in solar units. We expect that the luminosities of the components of SB2 systems are less accurate than for the single stars as a result of the more complex way the information was extracted from the data.

The Hertzsprung-Russell (HR) diagram of our targets is displayed in Fig. 8 where we overplot PARSEC isochrones at $Z=0.021([\mathrm{Fe} / \mathrm{H}]=+0.14$, see Sect. 5.3) at three ages. As can be seen, most of the targets are located in the region of the HR diagram where the MS isochrones overlap, so that very little information about the cluster age can be gathered from this plot.
The position of the two components of S 56 is significantly above the above-mentioned isochrones, suggesting that these stars are more evolved (older age) or that some mass exchange has occurred between the components of the system. However, as outlined in Sect. 5.2, the parameters of the components of this system are very uncertain and should be taken with caution.

The position of the hottest source in our sample, S 466, is in between the $155 \mathrm{Myr}$ and $250 \mathrm{Myr}$ isochrones. This is in line with the CMDs of Fig. 3 in which it (upper red dot) is slightly displaced redward with respect to the $155 \mathrm{Myr}$ isochrone. However, the hottest member of the cluster, HD 216057, seems to fit well on the $155 \mathrm{Myr}$ isochrone in CMDs (upper gray dot in each panel of Fig. 3).

\section{Chromospheric emission and lithium abundance}

For stars with an age from a few ten to a few hundred Myr, the $\mathrm{H} \alpha$ emission (diagnostic of chromospheric activity) and lithium absorption can be used to estimate the age (see, e.g., Jeffries 2014; Frasca et al. 2018, and references therein).

To this end, the synthetic spectra produced by ROTFIT and COMPO2 with non-active, lithium-poor templates were subtracted from the observed spectra of the targets to measure the excess emission in the core of the $\mathrm{H} \alpha$ line $\left(\mathrm{EW}_{\mathrm{H} \alpha}^{\mathrm{em}}\right)$ and the equivalent width of the Li I $\lambda 6708 \AA$ absorption line $\left(\mathrm{EW}_{\mathrm{Li}}\right)$, removing the blends with nearby lines.

An example of spectral subtraction for a single-lined spectrum is shown in Fig. 9. The values of $\mathrm{EW}_{\mathrm{H} \alpha}^{\mathrm{em}}$ and $\mathrm{EW}_{\mathrm{Li}}$ were measured on the subtracted spectra by integrating the residual emission and absorption profiles, respectively, and are quoted in Table 6. For SB2 systems, subtraction is even more necessary to remove different lines of the two components which, due to the different Doppler shifts, overlap one another. The difference spectrum contains only the Li I absorption lines of the two components, whose equivalent widths can be measured individually by means of a two-Gaussian deblending (see Fig. 10 for an example). We note that the values of $\mathrm{EW}_{\mathrm{Li}}$ for the components of SB2 systems, which are quoted in Table 6, were corrected for the contribution to the continuum of the respective component.

Lithium is a fragile element that is burned in stellar interiors at temperature as low as $2.5 \times 10^{6} \mathrm{~K}$. It is progressively depleted from the stellar atmosphere in a way depending on the internal structure (i.e., stellar mass) for stars with convective envelopes deep enough to reach temperatures at which lithium is burned. It can therefore be used as an age proxy for stars cooler than about $6500 \mathrm{~K}$.

A simple way to get an estimate of stellar ages is a diagram that shows $\mathrm{EW}_{\mathrm{Li}}$ or lithium abundance, $A(\mathrm{Li})$, versus $T_{\text {eff }}$ for the investigated stars and to compare their positions with the upper envelopes of clusters with a known age. The isochronal age estimated for ASCC 123 in the literature ranges from about $130 \mathrm{Myr}$ (Yen et al. 2018) to $260 \mathrm{Myr}$ (Kharchenko et al. 2005). The CMDs of the present paper (Fig. 3) do not allow us to notably improve the age determination, which is $\tau \approx 150 \mathrm{Myr}$ with an uncertainty of at least $50 \mathrm{Myr}$. This is basically due to the lack of turn-off cluster members, as mentioned above. For this reason and to identify anomalous or contaminant stars in our sample, we used as comparison the upper envelopes of the following clusters: the Hyades (Soderblom et al. 1990), the Pleiades (Soderblom et al. 1993a; Neuhäuser et al. 1997), and IC 2602 (Montes et al. 2001), whose ages are of about $650 \mathrm{Myr}, 125 \mathrm{Myr}$ (White et al. 2007), and 30 Myr (Stauffer et al. 1997), respectively. As shown by Soderblom et al. (1993b), the lower envelope 

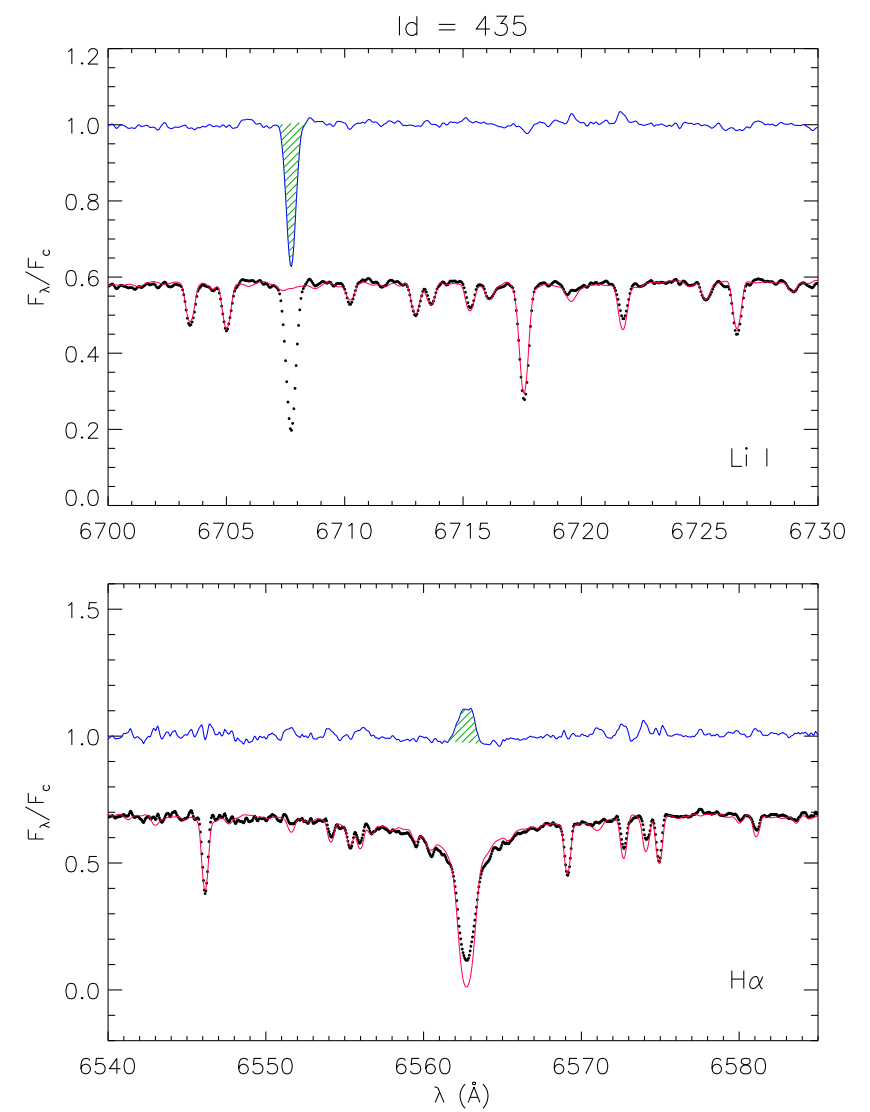

Fig. 9. Subtraction of the non-active, lithium-poor template (red line) from the spectrum of S 435 (black dots), which reveals the chromospheric emission in the $\mathrm{H} \alpha$ core (blue line in the bottom panel) and emphasizes the LiI $\lambda 6708 \AA$ absorption line, removing the nearby blended lines (top panel). The green hatched areas represent the excess $\mathrm{H} \alpha$ emission (bottom panel) and Li I absorption (top panel) that were integrated to obtain $W_{\mathrm{H} \alpha}^{\mathrm{em}}$ and $W_{\mathrm{Li}}$, respectively.

of the Pleiades behaves as an upper boundary for the members of the Ursa Major (UMa) cluster ( $\tau \approx 300 \mathrm{Myr}$ ).

The $T_{\text {eff }}-\mathrm{EW}_{\mathrm{Li}}$ diagram is shown in Fig. 11. According to the range of isochronal ages, the $\mathrm{EW}_{\mathrm{Li}}$ values should be comprised between the lower and upper envelope of the Pleiades cluster.

We note that the $\mathrm{EW}_{\mathrm{Li}}$ value for $\mathrm{S} 490$ is too low, which is totally inconsistent with the cluster age. This reinforces the hypothesis that this star does not belong to ASCC 123, as already suggested by its kinematics. Both components of the binary system $\mathrm{S} 502$ also display low $\mathrm{EW}_{\mathrm{Li}}$ values that are compatible with an older age $(\tau \approx 600 \mathrm{Myr})$. For this reason, we do not consider S 502 as a bona fide member of the cluster. The other stars and systems instead display higher $\mathrm{EW}_{\mathrm{Li}}$ values, with the exception of the secondary components of S 708 and S 492. However, the primary components of these two systems show $\mathrm{EW}_{\mathrm{Li}}$ values compatible with a young age. We note that EW measurement is a more difficult task for binary system components since the contribution to the continuum also plays a role. This could affect the weaker components more. Nevertheless, we cannot exclude that binarity may have an effect on the depletion of lithium.

We note that four objects lie above the upper envelope of the Pleiades and below that of IC 2602, suggesting an age younger than $100 \mathrm{Myr}$ or an age spread. However, the two early Ktype members display a lithium content that is in very good agreement with the Pleiades. To answer these issues, high- or
Table 6. $\mathrm{H} \alpha$, Li I $26708 \AA$ equivalent widths and lithium abundance for the single/SB1 systems and the components of SB2 systems.

\begin{tabular}{|c|c|c|c|c|c|c|c|c|}
\hline \multirow[t]{2}{*}{ ID } & \multirow{2}{*}{$\begin{array}{l}T_{\text {eff }} \\
(\mathrm{K}) \\
\end{array}$} & $\underline{\mathrm{EW}_{\mathrm{H} \alpha}^{\mathrm{em}}}$ & Err & \multirow[t]{2}{*}{$R_{\mathrm{H} \alpha}^{\prime}$} & $\underline{\mathrm{EW}_{\mathrm{Li}}}$ & \multirow[t]{2}{*}{ Err } & $A(\mathrm{Li})$ & \multirow[t]{2}{*}{ Err } \\
\hline & & \multicolumn{2}{|c|}{$(\mathrm{m} \AA)$} & & $(\mathrm{m} \AA)$ & & (dex) & \\
\hline 39 & 6667 & 73 & 13 & $-5.09_{-0.09}^{+0.07}$ & 59 & 8 & $2.97^{(a)}$ & 0.08 \\
\hline 214 & 5804 & 291 & 21 & $-4.46_{-0.04}^{+0.04}$ & 211 & 14 & 3.42 & 0.19 \\
\hline 435 & 5758 & 144 & 18 & $-4.76_{-0.06}^{+0.06}$ & 193 & 6 & 3.24 & 0.12 \\
\hline 490 & 6128 & 24 & 11 & $-5.55_{-0.27}^{+0.170}$ & 7 & 3 & 1.72 & 0.33 \\
\hline 517 & 5784 & 591 & 33 & $-4.15_{-0.03}^{+0.03}$ & 223 & 16 & 3.47 & 0.20 \\
\hline 554 & 6871 & $\ldots$ & $\ldots$ & $\ldots$ & 60 & 9 & $2.98^{(a)}$ & 0.08 \\
\hline 565 & 6683 & $\ldots$ & $\ldots$ & $\ldots$ & 63 & 4 & $3.00^{(a)}$ & 0.04 \\
\hline $56 \mathrm{P}$ & 6670 & $\ldots$ & $\ldots$ & $\ldots$ & 85 & 15 & $3.18^{(a)}$ & 0.11 \\
\hline $266 \mathrm{P}$ & 6094 & $\ldots$ & $\ldots$ & $\ldots$ & 106 & 4 & 3.00 & 0.08 \\
\hline $266 \mathrm{~S}$ & 5550 & $\ldots$ & $\ldots$ & $\ldots$ & 182 & 12 & 2.95 & 0.30 \\
\hline $492 \mathrm{P}$ & 6075 & $136^{(b)}$ & 11 & $\ldots$ & 111 & 6 & 3.01 & 0.09 \\
\hline $492 \mathrm{~S}$ & 5558 & $\ldots$ & $\ldots$ & $\ldots$ & 73 & 12 & 2.26 & 0.28 \\
\hline $502 \mathrm{P}$ & 5730 & $64^{(b)}$ & 9 & $\ldots$ & 70 & 11 & 2.41 & 0.20 \\
\hline $502 \mathrm{~S}$ & 5628 & $\ldots$ & $\ldots$ & $\ldots$ & 65 & 13 & 2.27 & 0.26 \\
\hline $708 \mathrm{P}$ & 6114 & $\ldots$ & $\ldots$ & $\ldots$ & 174 & 7 & 3.45 & 0.12 \\
\hline $708 \mathrm{~S}$ & 6099 & $\ldots$ & $\ldots$ & $\ldots$ & 58 & 25 & 2.64 & 0.39 \\
\hline F1 & 5263 & 196 & 22 & $-4.63_{-0.06}^{+0.05}$ & 163 & 9 & 2.51 & 0.16 \\
\hline $\mathrm{F} 2$ & 5237 & 396 & 30 & $-4.32_{-0.05}^{+0.04}$ & 213 & 10 & 2.79 & 0.16 \\
\hline
\end{tabular}

Notes. ${ }^{(a)} T_{\text {eff }}>6500 \mathrm{~K} . A(\mathrm{Li})$ extrapolated from the Soderblom et al. (1993a) tables. ${ }^{(b)}$ Blended $\mathrm{H} \alpha$ residual emission profile. The total residual emission has been integrated.

mid-resolution spectra of lower mass members, which are too faint for GIARPS, would be of great help.

We derived $A(\mathrm{Li})$ from $\mathrm{EW}_{\mathrm{Li}}, T_{\text {eff }}$ and $\log g$ by interpolating the curves of growth of Soderblom et al. (1993a), which were preferred to those of Pavlenko \& Magazzù (1996) because the former encompass the $T_{\text {eff }}$ range $4000-6500 \mathrm{~K}$, while the latter, although calculated in non-LTE (NLTE), end at $6000 \mathrm{~K}$. However, the differences of $A(\mathrm{Li})$ for the stars with $T_{\text {eff }} \leq 6000 \mathrm{~K}$ are at most 0.15 dex. The values of $A(\mathrm{Li})$ are listed in Table 6 .

For the $\mathrm{H} \alpha$ line, we detected a relevant filling in the line core for all the three G-type stars. Two of them, S 214 and S 517, are ultrafast rotators, with $v \sin i=80-100 \mathrm{~km} \mathrm{~s}^{-1}$, while $\mathrm{S} 435$ rotates more slowly $\left(v \sin i \simeq 12 \mathrm{~km} \mathrm{~s}^{-1}\right)$, but still displays a $\mathrm{H} \alpha$ core filling.

For these stars we computed the $\mathrm{H} \alpha$ surface flux, $F_{\mathrm{H} \alpha}$, and the ratio of the $\mathrm{H} \alpha$ to bolometric luminosity, $R_{\mathrm{H} \alpha}^{\prime}$ as

$F_{\mathrm{H} \alpha}=F_{6563} W_{\mathrm{H} \alpha}^{\mathrm{em}}$,

$R_{\mathrm{H} \alpha}^{\prime}=L_{\mathrm{H} \alpha} / L_{\mathrm{bol}}=F_{\mathrm{H} \alpha} /\left(\sigma T_{\mathrm{eff}}^{4}\right)$,

where $F_{6563}$ is the flux at the continuum near the $\mathrm{H} \alpha$ line per unit stellar surface area, which is evaluated from the NextGen synthetic low-resolution spectra (Hauschildt et al. 1999) at the stellar temperature and surface gravity of the target. We evaluated the flux error by taking into account the error of $W_{\mathrm{H} \alpha}^{\mathrm{em}}$ and the uncertainty in the continuum flux at the line center, $F_{6563}$, which is estimated considering the errors of $T_{\text {eff }}$ and $\log g$.

The index $R_{\mathrm{H} \alpha}^{\prime}$ is plotted against $T_{\text {eff }}$ in Fig. 12 along with the data for the Pleiades stars from Soderblom et al. (1993c), the UMa-cluster member HD 111456 (Freire Ferrero et al. 2004), and three young Sun-like stars in the field, namely V889 Her ( $\tau \approx 50 \mathrm{Myr}$, Frasca et al. 2010), KIC 7985370, and KIC 7765135 ( $\tau=100-200 \mathrm{Myr}$, Fröhlich et al. 2011). The chromospheric emission of the members of ASCC 123 is comparable with that of the Pleiades and young Sun-like stars, confirming that the former are indeed young stars ( $\tau=50-200 \mathrm{Myr})$, but cannot provide us with absolute ages. However, it is worth noting that for the three Sun-like targets $R_{\mathrm{H} \alpha}^{\prime}$ and $\mathrm{EW}_{\mathrm{Li}}$ are correlated: the more active the star, the higher the lithium abundance. 

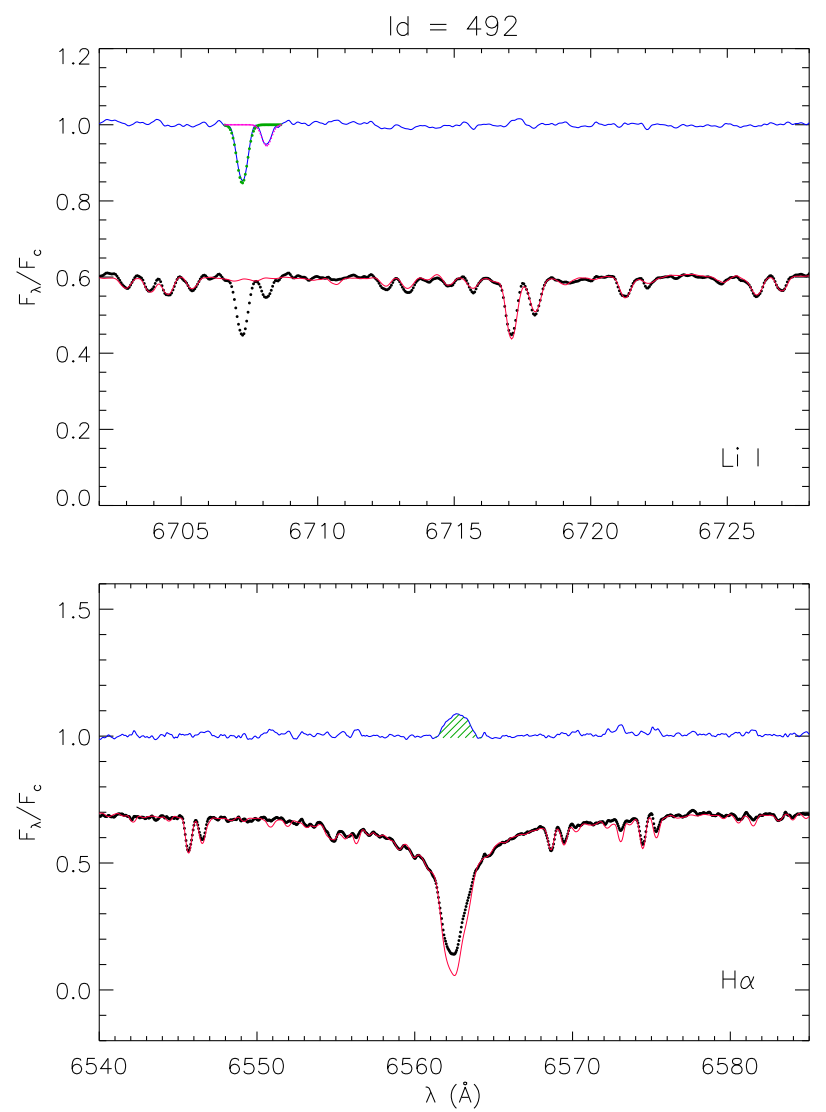

Fig. 10. Subtraction of the synthetic composite spectrum (red line) from the observed spectrum of the SB2 S 492 (black dots), which leaves only the chromospheric emission in the $\mathrm{H} \alpha$ core (blue line in the bottom panel) and emphasizes the LiI $\lambda 6708 \AA$ absorption lines of the two components (blue line in the top panel). The green and magenta dotted lines superimposed on the residual spectrum represent Gaussian fits used to deblend the Li I lines of the two components. The two components cannot be separated in the $\mathrm{H} \alpha$, and the full residual $\mathrm{H} \alpha$ emission can be integrated to provide a total $W_{\mathrm{H} \alpha}^{\mathrm{em}}$ (green hatched area in the bottom panel).

\section{Discussion}

We performed a comprehensive study of the cluster ASCC 123 resorting to different approaches and datasets, such as Gaia astrometry, photometry, and high-resolution spectroscopy. All together these data allow us to better define the properties of this cluster.

The atmospheric parameters, particularly $T_{\text {eff }}$, along with the photometry allowed us to evaluate the average extinction towards the cluster as $A_{V} \simeq 0.13 \mathrm{mag}$, which implies a reddening $E(B-V) \simeq 0.04 \mathrm{mag}$ assuming $R_{V}=3.1$. This value is a little smaller than the value of $E(B-V) \simeq 0.10$ mag reported in the literature for this cluster (e.g., Kharchenko 2001; Yen et al. 2018). However, it is in agreement with the Gaia-2MASS 3D extinction maps of Lallement et al. (2019, see their Fig. A.1 at $\left.l=105^{\circ}\right)$.

We used different diagnostics to constrain the age of the cluster, namely the CMDs, the HR diagram, the chromospheric activity level, and the lithium abundance. The determination of extinction and metallicity has allowed us to select the proper isochrones to fit the CMDs and HR diagram. Unfortunately, the absence of turn-off stars prevents us from an accurate determination of the cluster age from the isochrones. However, an upper limit of $\tau \approx 250$ Myr can be defined. The lithium abundance

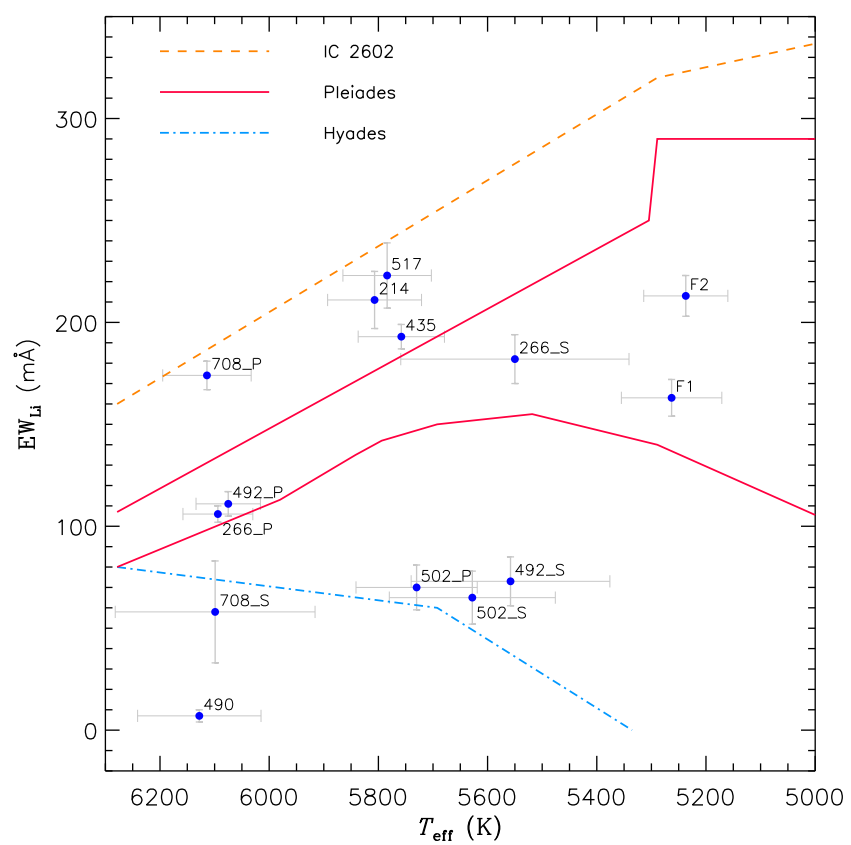

Fig. 11. Lithium equivalent width vs. effective temperature for the single stars and the components of SB2 systems. The upper envelopes (and lower envelope for the Pleiades) of a few young clusters are overplotted. The points are labeled with the star ID, as listed in Table 6 .

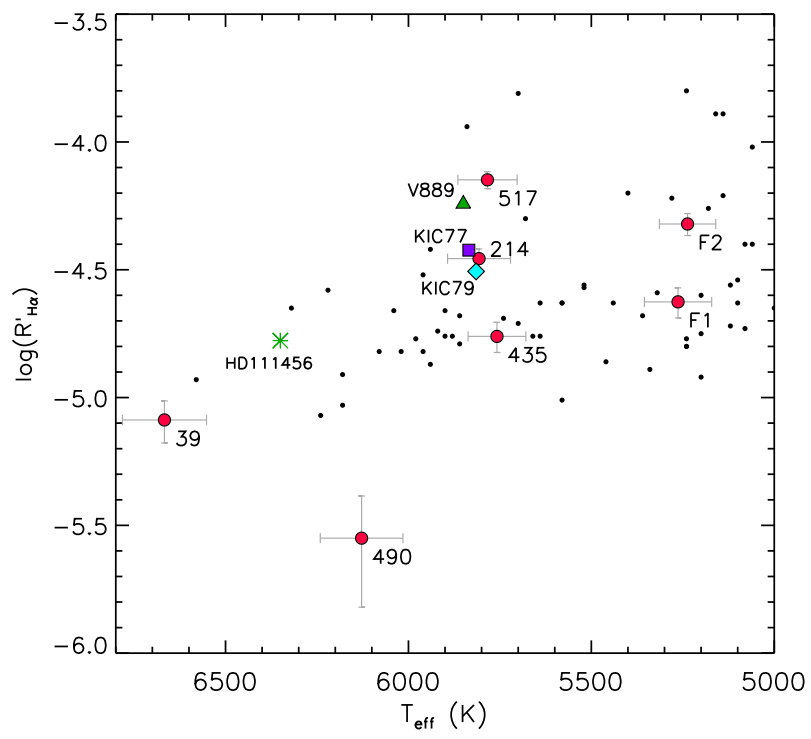

Fig. 12. Index $R_{\mathrm{H} \alpha}^{\prime}$ as a function of $T_{\mathrm{eff}}$ for the single-lined objects (red dots). The stars are labeled according to their IDs. The small black dots denote the measures made by Soderblom et al. (1993c) for Pleiades stars. The triangle refers to V889 Her (Frasca et al. 2010), while the diamond and square denote the two Sun-like stars KIC 7985370 and KIC 7765135, respectively (Fröhlich et al. 2011). The asterisk refers to the young F-type star HD 111546 (Freire Ferrero et al. 2004).

and the $\mathrm{H} \alpha$ flux suggest an age similar to that of the Pleiades ( $\tau \simeq 125 \mathrm{Myr}$ ) or a little younger, but certainly not older than the UMa cluster $(\tau \simeq 300 \mathrm{Myr})$. Therefore, we can only infer an age range of 100-250 Myr.

As already commented in Sect. 1, this is the first time that a chemical analysis has been performed on ASCC 123. In the absence of a previous study to use for a comparative discussion of our results, we resorted to the Galactic gradient as a 


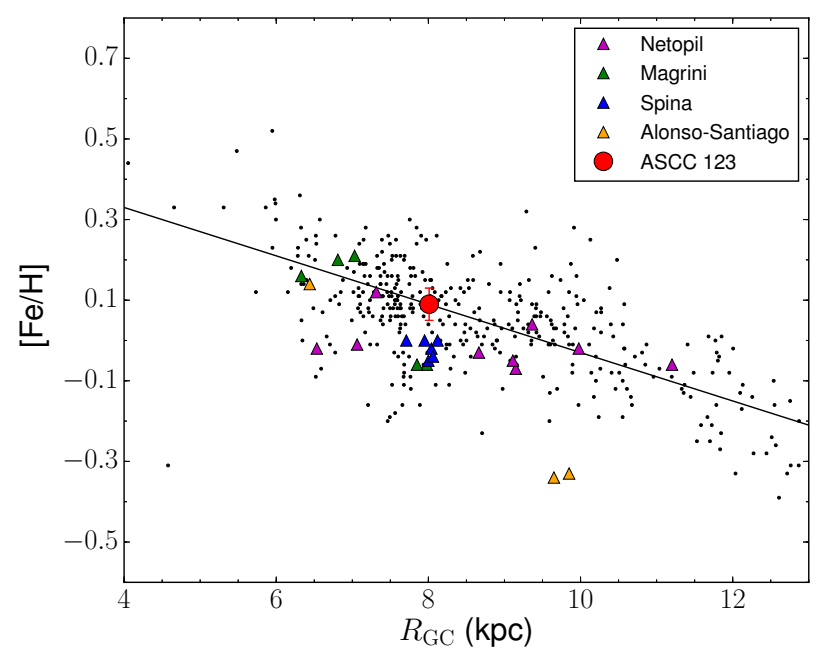

Fig. 13. Iron abundance gradient in the Milky Way found by Genovali et al. $(2013,2014)$ based on Cepheids (small black dots). The black line is the Galactic gradient $\left(-0.06 \mathrm{dex} \mathrm{kpc}^{-1}\right)$. Triangles represent young open clusters ( $<500 \mathrm{Myr}$ ) studied by Netopil et al. (2016), Spina et al. (2017), Magrini et al. (2018), and Alonso-Santiago et al. (2017, 2018, 2019). Finally, the red circle is ASCC 123. All the values shown in this plot are rescaled to Genovali et al. (2014) values: $R_{\odot}=7.95 \mathrm{kpc}$ and $A(\mathrm{Fe})=7.50$.

reference for a proper comparison. Genovali et al. (2013, 2014) traced the distribution of the metallicity in the Galactic disk, in terms of $[\mathrm{Fe} / \mathrm{H}]$, as a function of the Galactocentric distance. For this task they used the Cepheids, with ages between 20 and $400 \mathrm{Myr}$, which makes them very suitable for comparison to young OCs. In Fig. 13 ASCC 123 is overplotted on this gradient. Other young clusters $(\tau \leq 500 \mathrm{Myr})$ taken from Netopil et al. (2016), Spina et al. (2017), Magrini et al. (2018), and Alonso-Santiago et al. (2017, 2018, 2019) were added for further comparison. We note that ASCC 123 (with an average $[\mathrm{Fe} / \mathrm{H}]=+0.14 \pm 0.04$ ) follows closely the average trend with the Galactocentric distance. This result might seem at odds with previous findings of a solar or sub-solar metallicity for young and intermediate-age clusters (see, e.g., D’Orazi et al. 2011; Spina et al. 2017, and references therein). However, it is in agreement with what is expected from standard chemical evolution (e.g., Minchev et al. 2013, and references therein) and recent results of a super-solar metallicity $([\mathrm{Fe} / \mathrm{H}] \approx+0.2)$ for some young or intermediate-age clusters in the solar neighborhood, such as NGC 6067 (Alonso-Santiago et al. 2017) and Praesepe (D’Orazi et al. 2019).

We also compare our abundances with the Galactic trends found in the solar neighborhood by the Gaia-ESO Survey for the thin and thick disks (data release 4$)^{3}$. In Figs. A.2 and A.3 we plot some abundance ratios $[\mathrm{X} / \mathrm{Fe}]$ vs. $[\mathrm{Fe} / \mathrm{H}]$. The chemical composition of ASCC 123 is generally in agreement with the Galactic trends observed in the thin disk of the Galaxy. A few elements (V, Co, and Y) seem to be slightly overabundant, but still compatible with the Galactic trends within the errors. Instead, $\mathrm{Na}$ and $\mathrm{Zn}$ appear slightly underabundant, but the discrepancy from the bulk of Gaia-ESO Survey data, at least for sodium, is $\sim 1 \sigma$ and therefore it is not significant. The underabundance of $\mathrm{Zn}$ could be marginally significant.

https://www.eso.org/qi/catalogQuery/index/121

\section{Summary}

In the framework of the SPA project, we studied a sample of 17 candidate members to the young open cluster ASCC 123. We performed, for the first time, a thorough analysis of their properties based on high-resolution spectroscopy with the aim of improving our knowledge of this nearby and very sparse cluster.

We measured their radial and projected rotational velocities and found five new SB2 systems. We derived their atmospheric parameters, chromospheric activity level and lithium abundance by means of our own tools. For the single stars we also carried out a detailed chemical analysis.

We found that 3 out of the 17 targets have to be considered as non-members on the basis of their astrometry and lithium content. From the likely single-lined members, which are all MS stars, we derived an average extinction $A_{V}=0.13 \mathrm{mag}$, a median $\mathrm{RV}=-5.6 \mathrm{~km} \mathrm{~s}^{-1}$, and an average iron abundance $[\mathrm{Fe} / \mathrm{H}]=+0.14$, which is in very good agreement with the Galactic metallicity gradient. We also infer a chemical composition of the cluster compatible with the solar neighborhood, as traced by the Gaia-ESO survey. For ASCC 123 only a few elements display abundance ratios slightly discrepant with respect to these average trends. Those with the largest differences are $\mathrm{Zn}$ (underabundant) and Y (overabundant).

The analysis of lithium content (for the stars with $T_{\text {eff }}<$ $6500 \mathrm{~K}$ ) shows that six targets have an abundance comparable to or even higher than that measured for the Pleiades, a nearly coeval cluster. The chromospheric activity level, as traced by the index $R_{\mathrm{H} \alpha}^{\prime}$, for these stars is also in line with that of the Pleiades and of young Sun-like stars. This data confirm the young age of ASCC 123 inferred from the color-magnitude diagrams, suggesting a range of about 100-250 Myr.

Acknowledgements. We thank the referee for the very useful comments and suggestions. We acknowledge the support from the Italian Ministero dell'Istruzione, Università e Ricerca (MIUR). This research made use of SIMBAD and VIZIER databases, operated at the CDS, Strasbourg, France. This work has made use of data from the European Space Agency (ESA) mission Gaia (https://www.cosmos.esa.int/gaia), processed by the Gaia Data Processing and Analysis Consortium (DPAC, https://www.cosmos.esa.int/ web/gaia/dpac/consortium). Funding for the DPAC has been provided by national institutions, in particular the institutions participating in the Gaia Multilateral Agreement. We made use of data from the Gaia-ESO Survey Data Archive, prepared and hosted by the Wide Field Astronomy Unit, Institute for Astronomy, University of Edinburgh, which is funded by the UK Science and Technology Facilities Council. This publication made use of data products from the Two Micron All Sky Survey, which is a joint project of the University of Massachusetts and the Infrared Processing and Analysis Center/California Institute of Technology, funded by the National Aeronautics and Space Administration and the National Science Foundation.

\section{References}

Alonso-Santiago, J., Negueruela, I., Marco, A., et al. 2017, MNRAS, 469, 1330 Alonso-Santiago, J., Marco, A., Negueruela, I., et al. 2018, A\&A, 616, A124 Alonso-Santiago, J., Negueruela, I., Marco, A., et al. 2019, A\&A, 631, A124 Altmann, M., Roeser, S., Demleitner, M., Bastian, U., \& Schilbach, E. 2017, A\&A, 600, L4

Baranne, A., Queloz, D., Mayor, M., et al. 1996, A\&AS, 119, 373

Bertelli Motta, C., Pasquali, A., Richer, J., et al. 2018, MNRAS, 478, 425 Bossini, D., Vallenari, A., Bragaglia, A., et al. 2019, A\&A, 623, A108 Bressan, A., Marigo, P., Girardi, L., et al. 2012, MNRAS, 427, 127 Cantat-Gaudin, T., Jordi, C., Vallenari, A., et al. 2018, A\&A, 618, A93 Catanzaro, G. 2013, A\&A, 550, A79

Catanzaro, G., Ripepi, V., Bernabei, S., et al. 2011, MNRAS, 411, 1167 Catanzaro, G., Ripepi, V., \& Bruntt, H. 2013, MNRAS, 431, 3258

Claudi, R., Benatti, S., Carleo, I., et al. 2017, Eur. Phys. J. Plus, 132, 364 Cosentino, R., Lovis, C., Pepe, F., et al. 2014, Proc. SPIE, 9147, 91478 Covino, E., Esposito, M., Barbieri, M., et al. 2013, A\&A, 554, A28 
Cox, A. N. 2000, Allen's Astrophysical Quantities, 4th edn. (New York: AIP Press and Springer-Verlag)

D’Orazi, V., Biazzo, K., \& Randich, S. 2011, A\&A, 526, A103

D’Orazi, V., Oliva, T., Bragaglia, A., et al. 2019, A\&A, accepted

ESA 1997, The Hipparcos and Tycho Catalogues, ESA SP, 1200

Eker, Z., Soydugan, F., Soydugan, E., et al. 2015, AJ, 149, 131

Droege, T. F., Richmond, M. W., Sallman, M. P., \& Creager, R. P. 2006, PASP, 118,1666

Frasca, A., Freire Ferrero, R., Marilli, E., \& Catalano, S. 2000, A\&A, 364, 179

Frasca, A., Guillout, P., Marilli, E., et al. 2006, A\&A, 454, 301

Frasca, A., Biazzo, K., Kővári, Zs., Marilli, E., \& Çakırlı, Ö. 2010, A\&A, 518, A48

Frasca, A., Biazzo, K., Lanzafame, A. C., et al. 2015, A\&A, 575, A4

Frasca, A., Guillout, P., Klutsch, A., et al. 2018, A\&A, 612, A96

Freire Ferrero, R., Frasca, A., Marilli, E., \& Catalano, S. 2004, A\&A, 413, 657

Friel, E. D. 1995, ARA\&A, 33, 381

Fröhlich, H.-E., Frasca, A., Catanzaro, G., et al. 2011, A\&A, 543, A146

Gaia Collaboration (Brown, A. G. A., et al.) 2018, A\&A, 616, A1

Genovali, K., Lemasle, B., Bono, G., et al. 2013, A\&A, 554, A132

Genovali, K., Lemasle, B., Bono, G., et al. 2014, A\&A, 566, A37

Hauschildt, P. H., Allard, F., \& Baron, E. 1999, ApJ, 512, 377

Henden, A. A., Templeton, M., Terrell, D., et al. 2015, APASS: The AAVSO Photometric All-Sky Survey - Data Release 9, AAS Meeting S225, 336.16

Jackson, R. J., Deliyannis, C. P., \& Jeffries, R. D. 2018, MNRAS, 476, 3245

Janes, K., \& Adler, D. 1982, ApJS, 49, 425

Jeffries, R. D. 2014, in The Ages of Stars, eds. Y. Lebreton, D. Valls-Gabaud, \& C. Charbonnel, EAS Pub. Ser., 65, 289

Jeffries, R. D., Jackson, R. J., Franciosini, E., et al. 2017, MNRAS, 464, 1456

Johnson, H. L., Hoag, A. A., Iriarte, B., et al. 1961, Lowell Obs. Bull., 5, 133

Junqueira, T. C., Chiappini, C., Lépine, J. R. D., et al. 2015, MNRAS, 449, 2336

Kharchenko, N. V. 2001, Kinematika i Fizika Nebesnykh Tel, 17, 409

Kharchenko, N. V., Piskunov, A. E., Röser, S., Schilbach, E., \& Scholz, R.-D 2005, A\&A, 440, 403

Kharchenko, N. V., Piskunov, A. E., Schilbach, E., Röser, S., \& Scholz, R.-D 2013, A\&A, 558, A53
Kurucz, R. L. 1993a, in IAU Colloq. 138: Peculiar versus Normal Phe-nomena in A-type and Related Stars, eds. M. M. Dworetsky, F. Castelli, \& R. Faraggiana (San Francisco: Astron. Soc. Pac.), ASP Conf. Ser., 44, 87

Kurucz, R. L. 1993b, Kurucz CD-ROM 13: ATLAS9 (Cambridge, USA: Smithsonian Astro-physical Observatory)

Kurucz, R. L., \& Avrett, E. H. 1981, SAO Special Report 391 (Cambridge, USA: Smithsonian Astrophysical Observatory)

Lallement, R., Babusiaux, C., Vergely, J. L., et al. 2019, A\&A, 625, A135

Lasker, B., Lattanzi, M. G., McLean, B. J., et al. 2008, AJ, 136, 735

Magrini, L., Spina, L., Randich, S., et al. 2018, A\&A, 617, A106

Maíz Apellániz, J., \& Weiler, M. 2018, A\&A, 619, A180

Minchev, I., Chiappini, C., \& Martig, M. 2013, A\&A, 558, A9

Monteiro, H., \& Dias, W. S. 2019, MNRAS, 487, 2385

Montes, D., López-Santiago, J., Fernández-Figueroa, M. J., \& Gálvez, M. C. 2001, A\&A, 379, 976

Netopil, M., Paunzen, E., Heiter, U., \& Soubiran, C. 2016, A\&A, 585, A150

Neuhäuser, R., Torres, G., Sterzik, M. F., \& Randich, S. 1997, A\&A, 325, 647

Oliva, E., Origlia, L., Maiolino, R., et al. 2012a, Proc. SPIE, 8446E, 3 TO

Oliva, E., Biliotti, V., Baffa, C., et al. 2012b, Proc. SPIE , 8453E, 2 TO

Origlia, L., Oliva, E., Baffa, C., et al. 2014, Proc. SPIE, 914461E, 1E0

Origlia, L., Dalessandro, E., Sanna, N., et al. 2019, A\&A, 629, A117

Pavlenko, Y. V., \& Magazzù, A. 1996, A\&A, 311, 061

Pecaut, M. J., \& Mamajek, E. E. 2013, ApJS, 208, 9

Pepe, F., Mayor, M., Galland, F., et al. 2002, A\&A, 388, 632

Skrutskie, M. F., Cutri, R. M., Stiening, R., et al. 2006, AJ, 131, 1163

Smiljanic, R., Korn, A. J., Bergemann, M., et al. 2014, A\&A, 570, A122

Soderblom, D. R., Oey, M. S., Johnson, D. R. H., \& Stone, R. P. S. 1990, AJ, 99, 595

Soderblom, D. R., Jones, B. F., Balachandran, S., et al. 1993a, AJ, 106, 1059

Soderblom, D. R., Pilachowski, C. A., Fedel, S. B., \& Jones, B. F. 1993b, AJ, 105, 2299

Soderblom, D. R., Stauffer, J. R., Hudon, J. D., \& Jones, B. F. 1993c, ApJS, 85, 315

Spina, L., Randich, S., Magrini, L., et al. 2017, A\&A, 601, A70

Stauffer, J. R., Hartmann, L. W., Prosser, C. F. et al. 1997, ApJ, 479, 776

White, R. J., Gabor, J. M., \& Hillenbrand, L. A. 2007, AJ, 133, 2524

Yen, H.-W., Zhao, B., Koch, P. M., et al. 2018, A\&A, 615, A58 


\section{Appendix A: Additional tables and figures}

Table A.1. Photometric data for the stars observed in this work.

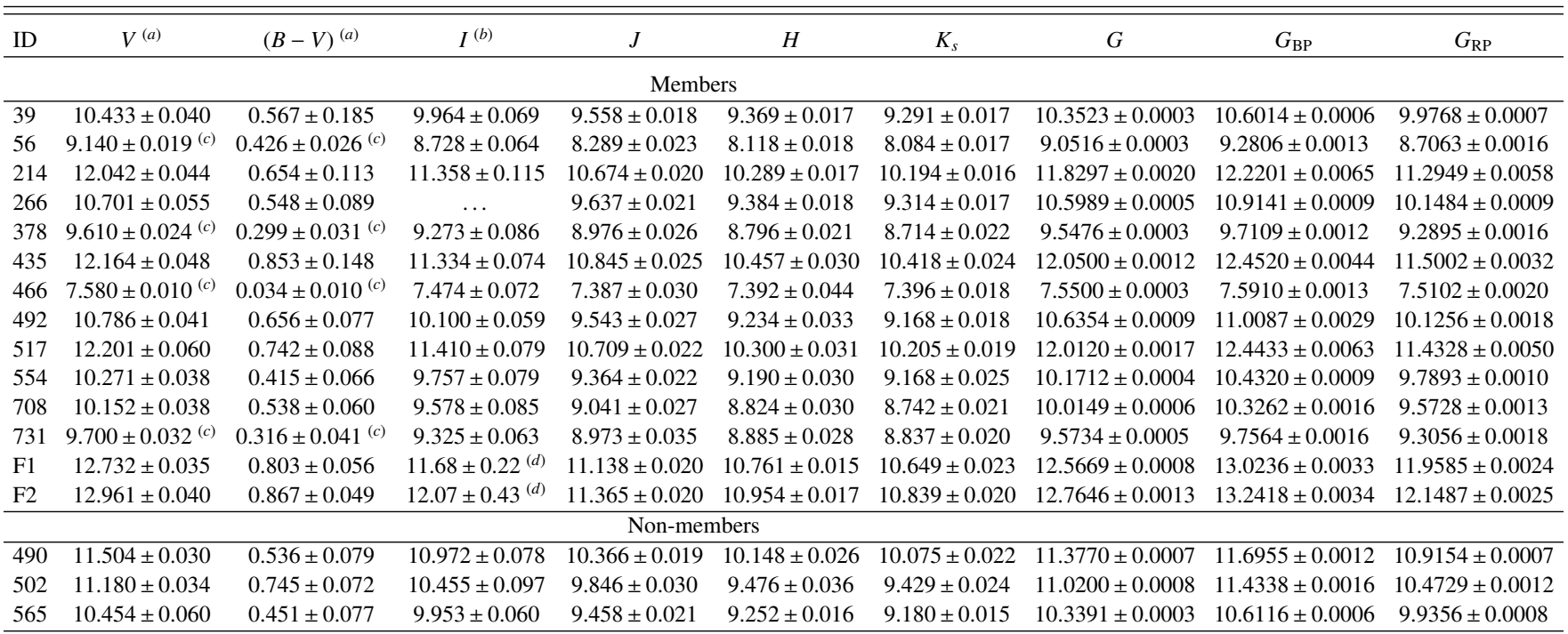

Notes. ${ }^{(a)}$ From the APASS catalog (Henden et al. 2015). ${ }^{(b)}$ From the TASS catalog (Droege et al. 2006). ${ }^{(c)}$ From the Tycho catalog (ESA 1997).

${ }^{(d)} N$ photographic magnitude from GSC2.3 catalog (Lasker et al. 2008).

Table A.2. Elemental abundances for the single/SB1 stars expressed according to the standard notation $A(X)=\log [n(X) / n(H)]+12$.

\begin{tabular}{|c|c|c|c|c|c|c|c|c|c|c|c|c|}
\hline Element & 39 & 214 & 378 & 435 & 466 & $490^{(*)}$ & 517 & 554 & $565^{(*)}$ & 731 & $\mathrm{~F} 1$ & $\mathrm{~F} 2$ \\
\hline $\mathrm{C}$ & $\ldots$ & & $8.49 \pm 0.15$ & & $8.22 \pm 0.13$ & $8.07 \pm 0.12$ & & $8.44 \pm 0.12$ & $8.37 \pm 0.14$ & $8.50 \pm 0.12$ & & \\
\hline $\mathrm{O}$ & $\ldots$ & $\ldots$ & $8.50 \pm 0.21$ & $\ldots$ & $8.81 \pm 0.17$ & & $\ldots$ & $9.82 \pm 0.15$ & $8.75 \pm 0.15$ & $8.79 \pm 0.18$ & & \\
\hline $\mathrm{Mg}$ & $7.76 \pm 0.19$ & $7.76 \pm 0.08$ & $8.06 \pm 0.12$ & $7.68 \pm 0.04$ & $7.59 \pm 0.06$ & $7.25 \pm 0.16$ & $7.79 \pm 0.11$ & $7.78 \pm 0.11$ & $7.50 \pm 0.15$ & $7.76 \pm 0.10$ & $7.61 \pm 0.18$ & $7.80 \pm 0.09$ \\
\hline $\mathrm{Al}$ & $\ldots$ & & $6.61 \pm 0.50$ & $6.23 \pm 0.15$ & $\ldots$ & $6.14 \pm 0.10$ & $\ldots$ & & $6.96 \pm 0.15$ & $6.40 \pm 0.12$ & $6.58 \pm 0.09$ & $6.52 \pm 0.15$ \\
\hline $\mathrm{Si}$ & $7.59 \pm 0.18$ & $7.18 \pm 0.14$ & $7.79 \pm 0.18$ & $7.35 \pm 0.10$ & $7.27 \pm 0.17$ & $7.00 \pm 0.18$ & $7.34 \pm 0.20$ & $7.52 \pm 0.10$ & $7.40 \pm 0.19$ & $7.82 \pm 0.13$ & $7.68 \pm 0.10$ & $7.78 \pm 0.19$ \\
\hline $\mathrm{S}$ & $7.33 \pm 0.13$ & $\ldots$ & $7.51 \pm 0.11$ & $\ldots$ & $\ldots$ & $7.07 \pm 0.10$ & $\ldots$ & $7.41 \pm 0.17$ & $7.13 \pm 0.10$ & $7.41 \pm 0.08$ & $\ldots$ & \\
\hline $\mathrm{Ca}$ & $6.54 \pm 0.13$ & $6.75 \pm 0.10$ & $6.63 \pm 0.09$ & $6.59 \pm 0.17$ & $6.46 \pm 0.19$ & $6.08 \pm 0.14$ & $6.65 \pm 0.13$ & $6.44 \pm 0.13$ & $6.36 \pm 0.16$ & $6.56 \pm 0.13$ & $6.49 \pm 0.12$ & $6.71 \pm 0.11$ \\
\hline $\mathrm{Sc}$ & $3.64 \pm 0.15$ & $3.66 \pm 0.16$ & $3.50 \pm 0.18$ & $3.42 \pm 0.16$ & & $2.67 \pm 0.10$ & $3.63 \pm 0.15$ & $3.67 \pm 0.10$ & $3.07 \pm 0.14$ & $3.50 \pm 0.13$ & $3.41 \pm 0.08$ & $3.39 \pm 0.12$ \\
\hline $\mathrm{Ti}$ & $4.95 \pm 0.17$ & $4.96 \pm 0.18$ & $5.14 \pm 0.12$ & $5.00 \pm 0.16$ & $4.26 \pm 0.19$ & $4.57 \pm 0.12$ & $4.95 \pm 0.16$ & $5.09 \pm 0.11$ & $4.86 \pm 0.11$ & $5.00 \pm 0.10$ & $5.01 \pm 0.10$ & $5.10 \pm 0.13$ \\
\hline V & $4.41 \pm 0.14$ & $4.53 \pm 0.15$ & $4.91 \pm 0.14$ & $4.20 \pm 0.12$ & $\ldots$ & $3.74 \pm 0.18$ & $4.65 \pm 0.14$ & $5.06 \pm 0.19$ & $4.43 \pm 0.15$ & $5.13 \pm 0.11$ & $4.07 \pm 0.10$ & $4.23 \pm 0.10$ \\
\hline $\mathrm{Cr}$ & $5.85 \pm 0.16$ & $5.67 \pm 0.14$ & $5.81 \pm 0.16$ & $5.81 \pm 0.10$ & $5.63 \pm 0.15$ & $5.35 \pm 0.12$ & $5.57 \pm 0.17$ & $5.84 \pm 0.17$ & $5.77 \pm 0.11$ & $5.79 \pm 0.18$ & $5.75 \pm 0.10$ & $5.86 \pm 0.10$ \\
\hline $\mathrm{Mn}$ & $5.73 \pm 0.08$ & $5.33 \pm 0.16$ & $5.46 \pm 0.06$ & $5.56 \pm 0.16$ & & $5.09 \pm 0.11$ & $5.57 \pm 0.14$ & $5.69 \pm 0.19$ & $5.45 \pm 0.13$ & $5.57 \pm 0.19$ & $5.59 \pm 0.12$ & $5.67 \pm 0.14$ \\
\hline $\mathrm{Fe}$ & $7.50 \pm 0.14$ & $7.68 \pm 0.15$ & $7.76 \pm 0.18$ & $7.61 \pm 0.12$ & $7.69 \pm 0.11$ & $7.03 \pm 0.11$ & $7.53 \pm 0.11$ & $7.62 \pm 0.09$ & $7.34 \pm 0.11$ & $7.61 \pm 0.17$ & $7.47 \pm 0.13$ & $7.57 \pm 0.11$ \\
\hline Co & & $5.20 \pm 0.10$ & $7.26 \pm 2.72$ & $4.85 \pm 0.12$ & $\ldots$ & $4.78 \pm 0.12$ & $5.34 \pm 0.16$ & $5.34 \pm 0.17$ & $5.45 \pm 0.18$ & $6.09 \pm 0.08$ & $4.93 \pm 0.10$ & $5.03 \pm 0.12$ \\
\hline $\mathrm{Zn}$ & $\ldots$ & $\ldots$ & $\ldots$ & $4.42 \pm 0.10$ & $\ldots$ & $4.12 \pm 0.10$ & $3.95 \pm 0.18$ & $\ldots$ & $4.33 \pm 0.07$ & & $4.60 \pm 0.11$ & $4.55 \pm 0.16$ \\
\hline $\mathrm{Sr}$ & $\ldots$ & $\ldots$ & $\ldots$ & $3.49 \pm 0.12$ & $\ldots$ & $\ldots$ & $\ldots$ & $\ldots$ & $4.26 \pm 0.35$ & $\ldots$ & $3.51 \pm 0.15$ & $3.76 \pm 0.18$ \\
\hline $\mathrm{Y}$ & $2.61 \pm 0.08$ & $3.15 \pm 0.26$ & $2.33 \pm 0.13$ & $\ldots$ & $\ldots$ & $2.10 \pm 0.15$ & $3.78 \pm 0.15$ & $2.51 \pm 0.16$ & $2.50 \pm 0.15$ & $2.25 \pm 0.12$ & $2.72 \pm 0.17$ & $2.82 \pm 0.18$ \\
\hline $\mathrm{Zr}$ & $3.30 \pm 0.14$ & $3.19 \pm 0.10$ & $3.66 \pm 0.19$ & $2.82 \pm 0.08$ & $\ldots$ & $2.42 \pm 0.12$ & $3.13 \pm 0.15$ & $3.23 \pm 0.07$ & $3.00 \pm 0.11$ & $3.48 \pm 0.18$ & $3.13 \pm 0.12$ & $3.46 \pm 0.16$ \\
\hline
\end{tabular}

Notes. ${ }^{(*)}$ Non-member. 
A\&A 632, A16 (2019)
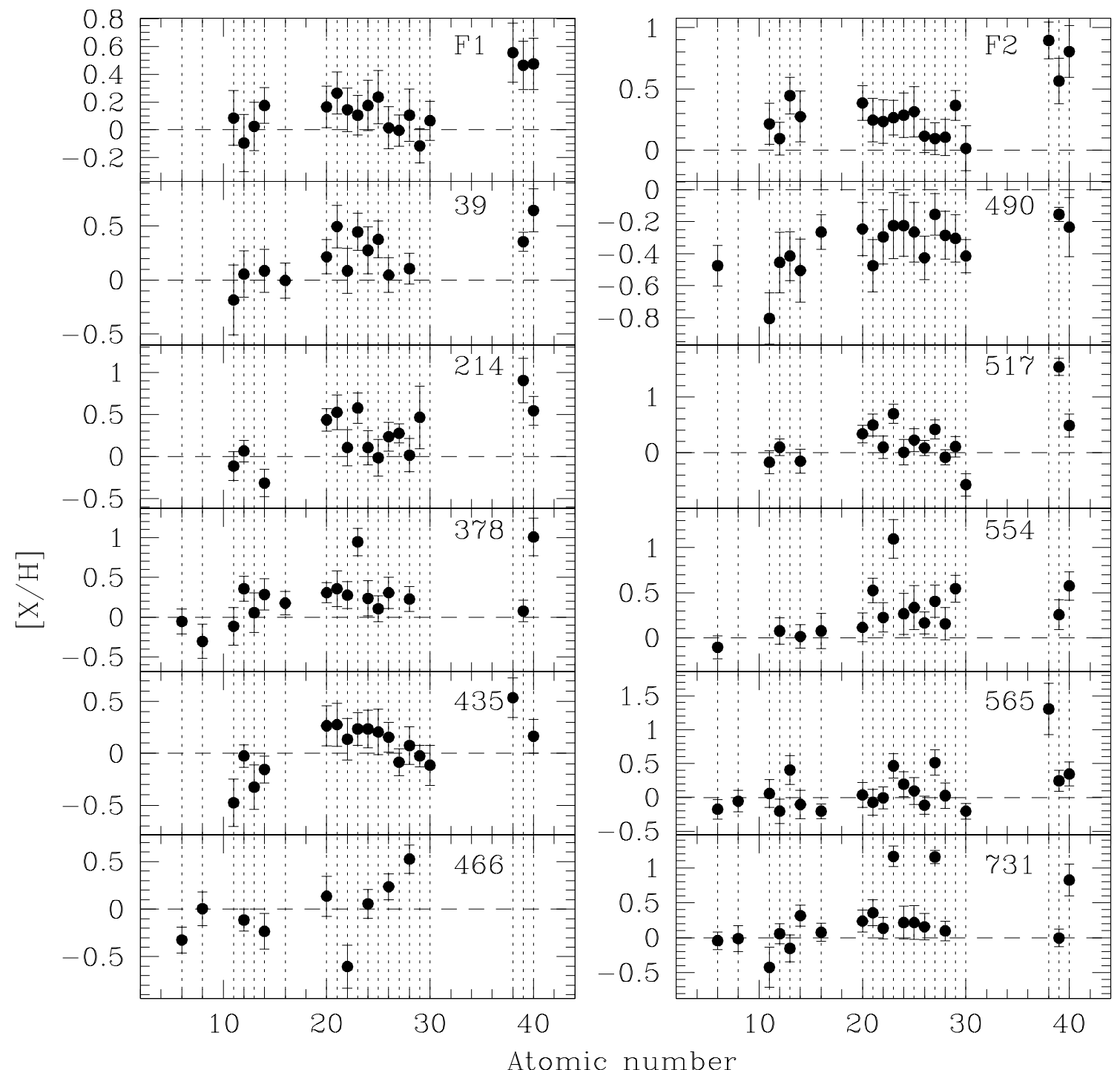

Fig. A.1. Chemical patterns derived for our targets. The horizontal dashed line in each box corresponds to the solar abundances reported in Table 5 . 

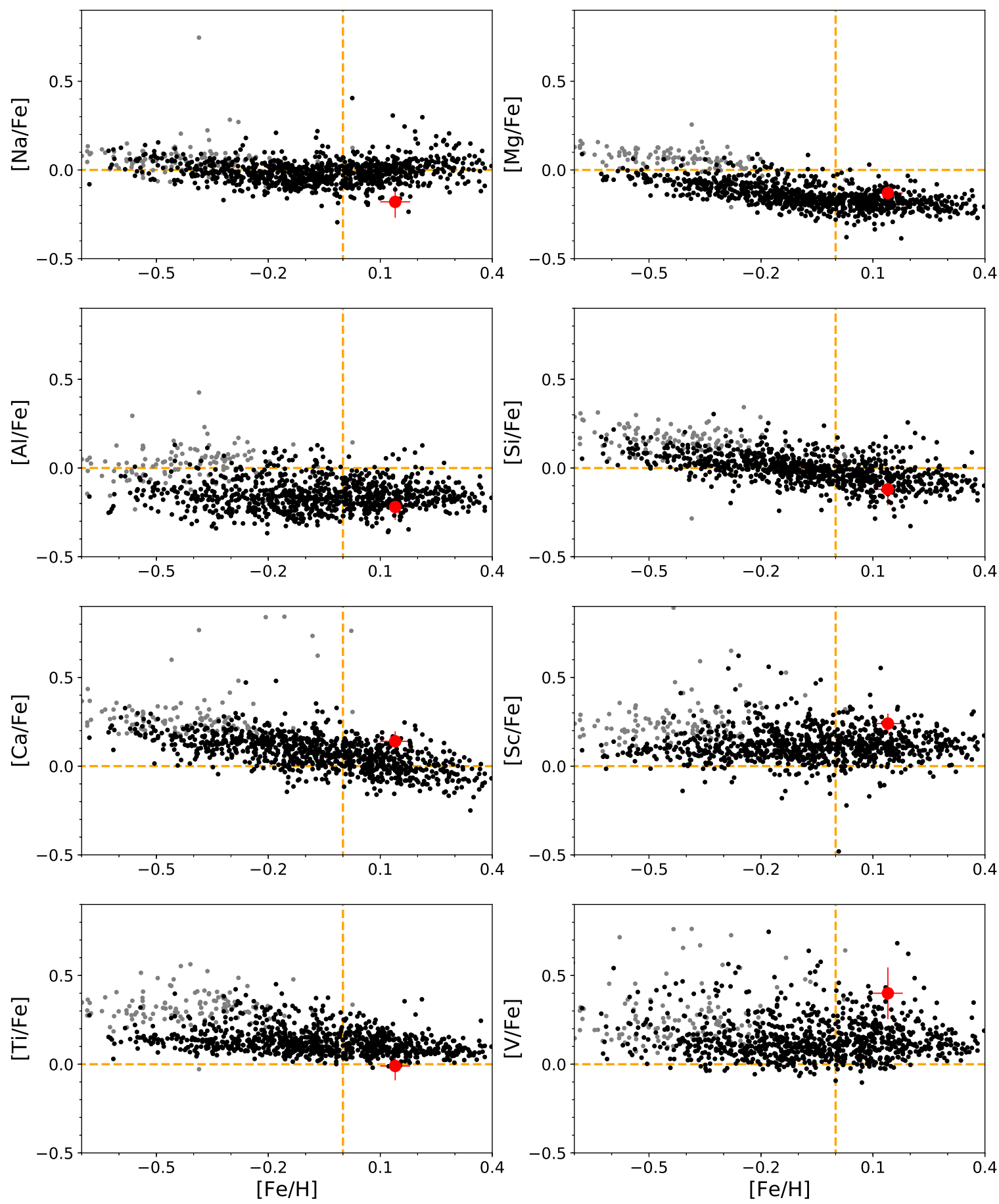

Fig. A.2. Abundance ratios $[\mathrm{X} / \mathrm{Fe}]$ vs. $[\mathrm{Fe} / \mathrm{H}]$. The red circle shows the average value for ASCC 123 and the error bars are the standard errors reported in Table 5. The gray and black dots represent the Galactic trends for the thick and thin disk, respectively, in the solar neighborhood from the internal data release 4 of the Gaia-ESO Survey. The dashed lines indicate the solar value. For all the elements the same scale is used for a proper comparison. 



Fig. A.3. Abundance ratios $[\mathrm{X} / \mathrm{Fe}]$ vs. $[\mathrm{Fe} / \mathrm{H}]$. Symbols are as in Fig. A.2. 